\title{
Extended lateral heating of the nighttime ionosphere by ground-based VLF transmitters
}

\author{
K. L. Graf, ${ }^{1}$ M. Spasojevic, ${ }^{1}$ R. A. Marshall, ${ }^{2}$ N. G. Lehtinen, ${ }^{1}$ \\ F. R. Foust, ${ }^{1}$ and U. S. Inan ${ }^{1,3}$ \\ Received 16 August 2013; revised 9 October 2013; accepted 11 November 2013; published 3 December 2013.
}

[1] The effects of ground-based very low frequency (VLF) transmitters on the lower ionosphere are investigated. Controlled modulation experiments are performed with the $21.4 \mathrm{kHz}, 424 \mathrm{~kW}$ VLF transmitter NPM in Lualualei, Hawaii, and physical effects of the NPM transmissions are studied with a subionospherically propagating VLF probe signal. Observed perturbations to the probe signal are consistent neither with expectations from transmitter-induced electron precipitation nor to off-path scattering from a concentrated heating region near the transmitter but rather appear to be the result of scattering from extended lateral heating of the ionosphere by the NPM transmitter. A large-scale computational modeling framework confirms theoretically that this form of ionospheric heating can account for the observed probe signal modulations, establishing that the lateral extent of ionospheric heating due to VLF transmitters is several thousand kilometers, significantly greater than previously recognized.

Citation: Graf, K. L., M. Spasojevic, R. A. Marshall, N. G. Lehtinen, F. R. Foust, and U. S. Inan (2013), Extended lateral heating of the nighttime ionosphere by ground-based VLF transmitters, J. Geophys. Res. Space Physics, 118, 7783-7797, doi:10.1002/2013JA019337.

\section{Introduction}

[2] Ground-based very low frequency (VLF, 3-30 kHz) transmitters are known to affect the Earth's ionosphere both directly through collisional heating [Inan, 1990] and indirectly through inducing the precipitation of energetic electrons from the Earth's radiation belts [Imhof et al., 1983]. At VLF frequencies, both the Earth and the ionosphere act as good conductors, mostly reflecting the incident electromagnetic wave and guiding its propagation to large distances around the globe. During each reflection from the ionosphere, collisional losses in the $D$ region attenuate the wave and heat the ionospheric electrons. This heating increases the electron-neutral collision frequency, modifying the medium's conductivity and creating an ionospheric disturbance which can perturb the propagation of additional electromagnetic waves. By detecting perturbations to a VLF probe signal propagating through the region, one can remotely sense the ionospheric disturbance and thus estimate the heating effect of the original VLF transmitter [Rodriguez et al., 1994]. While most of the energy radiated by a ground-based VLF transmitter remains guided

\footnotetext{
${ }^{1}$ Department of Electrical Engineering, Stanford University, Stanford, California, USA.

${ }^{2}$ Department of Aeronautics and Astronautics, Stanford University, Stanford, California, USA.

${ }^{3}$ Electrical Engineering Department, Koc University, Istanbul, Turkey.

Corresponding author: K. L. Graf, Space, Telecommunications and Radioscience Laboratory, Stanford University, 350 Serra Mall, Room 308, Stanford, CA 94305, USA. (graf@stanford.edu)

(C)2013. American Geophysical Union. All Rights Reserved. 2169-9380/13/10.1002/2013JA019337
}

between the Earth and the ionosphere and can contribute to ionospheric heating, approximately $20 \%$ penetrates through the ionosphere into the magnetosphere [Graf et al., 2013]. Once injected into the magnetosphere, VLF waves propagating in the whistler mode can induce the pitch angle scattering and precipitation of trapped energetic electrons [Inan, 1987]. Precipitating energetic electrons impinge upon the upper atmosphere, generating secondary ionization and an ionospheric disturbance which is typically shifted poleward in latitude from the VLF transmitter [Kulkarni et al., 2008]. This ionospheric disturbance, much like that of the direct ionospheric heating, can perturb the propagation of subionospheric VLF signals.

[3] The first recognized occurrence of ionospheric heating by radio waves was the so-called "Luxembourg" or "ionospheric cross-modulation" effect reported by Tellegen [1933], where the $252.1 \mathrm{kHz}$ Luxembourg broadcast was detected by radio receivers in Eindhoven, Holland cross modulated upon the $652.2 \mathrm{kHz}$ Beromünster, Switzerland broadcast. Luxembourg is located nearly along the line joining Beromünster and Eindhoven, and it was suggested that the Luxembourg effect was due to interaction between the two waves as they passed through the ionosphere [Bailey and Martyn, 1934; Bailey, 1938]. Huxley and Ratcliffe [1949] provided a survey of the early theoretical and experimental knowledge concerning ionospheric cross modulation, and Gurevich [1978] extended the theory while summarizing nonlinear phenomena in the ionosphere. Fejer [1970] analyzed the potential of applying this ionospheric cross-modulation effect to probe the lower ionosphere. Galejs [1972] outlined the ionospheric heating and crossmodulation theory specifically for VLF radio waves, with 
additional work on this topic by Ginzburg and Gurevich [1960] and Maslin [1975, 1976]. VLF waves typically reflect from the $D$ region of the nighttime ionosphere, and since radio waves tend to be most sensitive to ionospheric changes near their reflection height, VLF waves are uniquely well suited for probing the lower ionosphere. Barr et al. [1984] presented the effects of HF ionospheric heating on the subionospheric propagation of VLF waves, and Inan [1990] provided the first experimental evidence of ionospheric cross modulation with VLF transmitters. Taranenko et al. [1992, 1993] and Barr and Stubbe [1992] provided theoretical analysis of the phenomenon and a comparison between VLF and HF heating of the lower ionosphere. Inan et al. [1992], Rodriguez and Inan [1994], and Rodriguez et al. [1994] provided additional observations of VLF transmitter heating effects on subionospherically propagating VLF probe signals and showed the observations to be consistent with 3-D modeling of the VLF heating and probe signal perturbation. Rodriguez et al. [1994] showed that powerful, ground-based VLF transmitters in continual operation enhance the nearby ionospheric temperature by as much as a factor of 3 for a $1 \mathrm{MW}$ transmitter, with the enhancement most intense in an annular ring in the lower ionosphere within $150 \mathrm{~km}$ radius of the transmitter. Rodriguez and Inan [1994] showed that the heating leads to an electron density depletion of up to $30 \%$ due to an increase in the effective three-body electron attachment rate. While the heating and cooling rates are very rapid (on the order of microseconds and milliseconds, respectively), the electron density changes occur far more slowly (tens of seconds) [Glukhov et al., 1992; Rodriguez and Inan, 1994]. Much of the additional attention given to ionospheric heating by radio waves concerns the use of HF waves in ELF/VLF generation [e.g., James, 1985; Rietveld et al., 1986; Moore et al., 2007; Cohen et al., 2010a; Jin et al., 2013]. Critical to the more advanced heating models were the electron cooling rates and studies of ionospheric chemistry [e.g., Schunk and Nagy, 1978; Tomko et al., 1980].

[4] The capability of ground-based VLF transmitters to induce the precipitation of radiation belt electrons has been well established by satellite observations [Vampola, 1977; Imhof et al., 1983; Koons et al., 1981]. Theoretical analysis attributed the phenomenon to gyroresonant pitch angle scattering of the trapped energetic electron population by whistler mode VLF waves [Inan, 1987, and references therein], and computational modeling efforts have served to theoretically quantify the effects using the best parameter estimates available at the time [Abel and Thorne, 1998a, 1998b; Kulkarni et al., 2008]. Marshall et al. [2010] showed the optical signature of transmitter-induced precipitation to be undetectable by modern instruments, leaving subionospheric VLF remote sensing as the only known method potentially capable of measuring transmitter-induced precipitation from the ground. Subionospheric VLF remote sensing has successfully detected electron precipitation due to lightning [e.g., Helliwell et al., 1973; Johnson et al., 1999; Peter and Inan, 2007; Cotts et al., 2011], but the perturbations associated with VLF transmitter-induced precipitation have been considerably less distinct [Inan et al., 2007; Graf et al., 2011]. A key factor affecting the efficiency with which ground-based VLF transmitters can induce energetic electron precipitation is the rate of transionospheric attenuation of VLF signals, a factor that has only recently been accurately ascertained and validated [Helliwell, 1965; Starks et al., 2008; Tao et al., 2010; Cohen and Inan, 2012; Cohen et al., 2012; Graf et al., 2013], meaning previous efforts to quantify the role played by ground-based VLF transmitters in shaping the Earth's radiation belts [e.g., Abel and Thorne, 1998a, 1998b; Kim et al., 2011] still warrant revisitation.

[5] The fact that both the ionospheric heating and the induced electron precipitation phenomena can produce ionospheric disturbances which would perturb a subionospherically propagating VLF probe signal has led to some confusion in analyzing experimental results. A series of Naval VLF transmitter keying experiments were conducted in recent years with the goal of better quantifying the effect of ground-based VLF transmitters on the Earth's radiation belts. In these experiments, the $21.4 \mathrm{kHz}$ transmitter NPM in Lualualei, Hawaii was modulated in periodic keying formats (e.g., $5 \mathrm{~s}$ on $/ 5 \mathrm{~s}$ off) while the $24.8 \mathrm{kHz}$ transmitter NLK in Jim Creek, Washington provided a continuous probe signal for the detection of induced ionospheric disturbances (see Figure 1). The VLF receiver MI was installed on Midway Atoll to detect the NLK probe signal propagating through the theoretical NPM precipitation region, and the received NLK-MI probe signal was analyzed for the presence of periodic perturbations matching those of the modulated NPM signal. Inan et al. [2007] initially reported the detection of induced electron precipitation during these experiments, showing that the periodic modulation of the NPM transmitter did induce the periodic perturbation of the NLK probe signal. They concluded that the observed perturbations were due to induced precipitation, in part because of the geometry of the detection network: the NLK-MI probe signal pathway propagates directly through the theoretical NPM precipitation region and is never closer than $1750 \mathrm{~km}$ to the NPM transmitter. At this large distance from the NPM transmitter, Inan et al. [2007] discarded off-path scattering from the NPM heating region as a possible link, and they did not consider the possibility of extended lateral heating by NPM affecting the NLK probe signal along its path.

[6] Following improved analysis of the NLK-MI probe signal from the NPM keying experiments, Graf et al. [2011] contradicted Inan et al. [2007] by demonstrating that the observations could not have been caused by induced electron precipitation. Improved signal processing significantly reduced the noise in the probe signal perturbation analysis, clearly highlighting the lack of onset delay in the observed perturbations. For midlatitude VLF transmitters, the onset delay between a VLF-transmitted pulse and the resultant induced electron precipitation impinging upon the upper atmosphere would be at least $0.2 \mathrm{~s}$ given the whistler mode VLF wave and energetic electron travel times involved [Inan et al., 1985]. Practically, the onset delay and rise time is more likely to be between 0.7 and $2.0 \mathrm{~s}$ considering lengthier propagation paths, multiple bounce interactions in the equatorial plane, and multiple atmospheric backscatter interactions in both hemispheres [Inan et al., 1985; Cotts et al., 2011]. Thus, the $<0.05 \mathrm{~s}$ onset delay and rise time observed by Graf et al. [2011] eliminated induced electron precipitation as a possible cause and contradicted the conclusions of Inan et al. [2007]. Graf et al. [2011] also provided laboratory test results of the MI receiver to eliminate the 


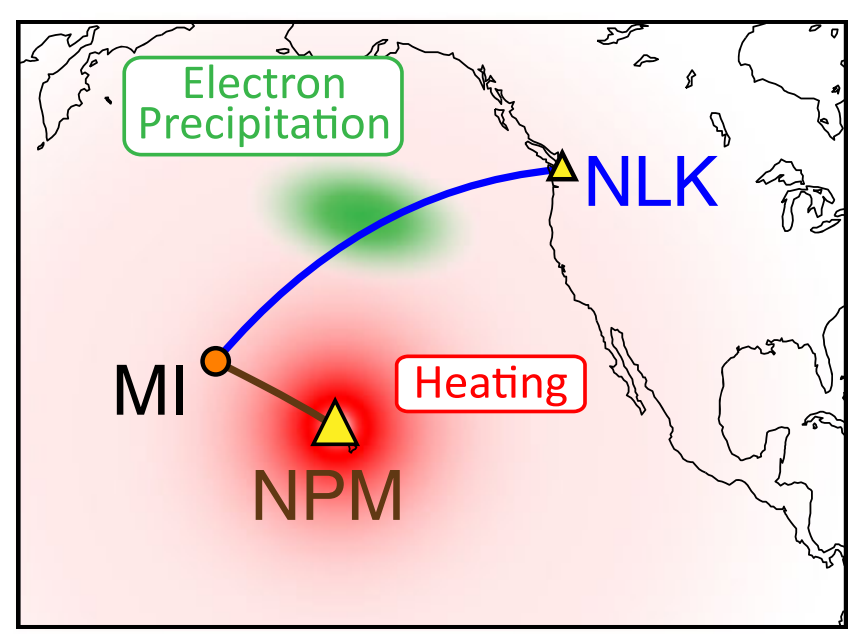

Figure 1. Map marking the location of VLF transmitters NPM in Lualualei, Hawaii, NLK in Jim Creek, Washington, and VLF receiver MI on Midway Atoll. The blue and brown lines denote the subionospheric great circle paths from the NLK and NPM transmitters to the MI receiver, respectively. Cartoon illustrations of hypothetical ionospheric disturbances due to NPM-induced ionospheric heating and electron precipitation are shaded in red and green, respectively. These are included for illustration purposes only and are not meant to indicate their relative intensity.

possibility of instrumental cross modulation in these keying experiments, leading to the conclusion that scattering from the NPM heating region was the most likely cause of the observed NLK-MI probe signal perturbations in the NPM keying experiments.

[7] In this paper, we extend the analysis of Graf et al. [2011] by combining additional experimental observations and data analysis with computational modeling to confirm theoretically that ionospheric heating can account for the observed probe signal modulations. We further show that the observed NLK-MI probe signal modulations are likely due to along-path scattering from extended lateral heating by NPM as opposed to off-path scattering from the more intense ionospheric heating near NPM. In the process, we develop a large-scale computational modeling framework for ionospheric heating by a ground-based VLF transmitter, and we show that the lateral extent of ionospheric heating due to VLF transmitters is several thousand kilometers significantly greater than previously recognized.

\section{VLF Transmitter Keying Experiments}

[8] The U.S. Navy's $400 \mathrm{~kW}, 21.4 \mathrm{kHz}$ VLF transmitter NPM located in Lualualei, Hawaii $\left(21.4^{\circ} \mathrm{N}, 158.2^{\circ} \mathrm{W}\right.$; $L=1.17$ ), was keyed on/off in periodic formats for two 30 min periods on most days from 25 August 2005 through 2 April 2008. The majority of the transmissions utilized a $0.1 \mathrm{~Hz}(5 \mathrm{~s}$ on $/ 5 \mathrm{~s}$ off) periodic keying format. A $5 \mathrm{~s}$ on $/ 5 \mathrm{~s}$ off format means NPM transmitted its $21.4 \mathrm{kHz}$ signal at nearly full power for $5 \mathrm{~s}$, then turned off for $5 \mathrm{~s}$, and repeated this cycle for the duration of the 30 min keying period. This controlled modulation is the key advantage of these experiments over the previous study of Rodriguez et al. [1994], where they were assessing the effects of single, fortuitous on/off transitions of VLF transmitters as opposed to their periodic modulation.

[9] The two-channel VLF receiver MI installed at Midway Atoll $\left(28.21^{\circ} \mathrm{N}, 177.38^{\circ} \mathrm{W}\right)$ provided ground-based VLF measurements of the local transverse magnetic field. This receiver is similar to the Atmospheric Weather Electromagnetic System for Observation, Modeling, and Education (AWESOME) instrument described by Cohen et al. [2010b]. The receiver features a pair of crossed, air-core, wire loop antennas for detecting the magnetic field in the north-south and east-west planes. Data are recorded at $100 \mathrm{kHz}$ sampling rate with 16 bits per sample, and the amplitude and phase of narrowband signals at specific frequency channels are demodulated in software and recorded at $50 \mathrm{~Hz}$. One of these narrowband frequency channels records the $24.8 \mathrm{kHz}$ signal from the $200 \mathrm{~kW}$ transmitter NLK in Jim Creek, Washington $\left(48.20^{\circ} \mathrm{N}, 121.92^{\circ} \mathrm{W}\right)$, while another records that of the keyed NPM transmitter. This provides a continuous probe signal for measuring ionospheric disturbances along the NLK-MI great circle path (GCP) from the transmitter NLK to the receiver MI. The geographic locations of NPM, NLK, and MI are marked in Figure 1, together with the GCPs of the NPM and NLK signals received at MI.

[10] Figure 1 also provides cartoon illustrations of theoretical ionospheric disturbances caused by NPM through direct ionospheric heating (in red) and induced electron precipitation (in green). Rodriguez et al. [1994] showed the ionospheric heating by a ground-based VLF transmitter to be strongest in an annular ring within $150 \mathrm{~km}$ of the transmitter. The dipole radiation pattern of a VLF transmitter produces a null directly overhead, and the most intense heating occurs at approximately $80 \mathrm{~km}$ radial distance. Heating is known to decrease at greater distances as the VLF wave energy spreads and attenuates, though Rodriguez et al. [1994] was interested primarily in the most intense portions of the heating region and thus only modeled the ionospheric heating to a radial extent of $250 \mathrm{~km}$. The cartoon heating region illustrated in red in Figure 1 is provided for illustrative purposes only, with its scale exaggerated to show the intense ring of heating near NPM and relatively weak heating extending laterally within the Earth-ionosphere waveguide to great distances. A quantitative analysis of the lateral extent and intensity of this heating region will be provided in section 3.2 .

[11] The location and extent of the ionospheric disturbance due to NPM-induced electron precipitation (in green) is based on the model of Kulkarni et al. [2008], where we note that the precipitation region is shifted poleward from NPM due to the nonducted, whistler mode propagation of the VLF waves within the magnetosphere. The precise location and intensity of this ionospheric disturbance is not definitively known following the recently updated transionospheric attenuation estimates of VLF waves [Cohen and Inan, 2012; Cohen et al., 2012; Graf et al., 2013] and a lack of experimental measurements for its quantification [ Graf et al., 2011]. Its existence and parameters will also be heavily dependent upon the pitch angle distribution of the trapped energetic electron population in the radiation belts [Graf et al., 2009]. Graf et al. [2011] concluded that the effect of this NPM-induced electron precipitation is below the threshold 


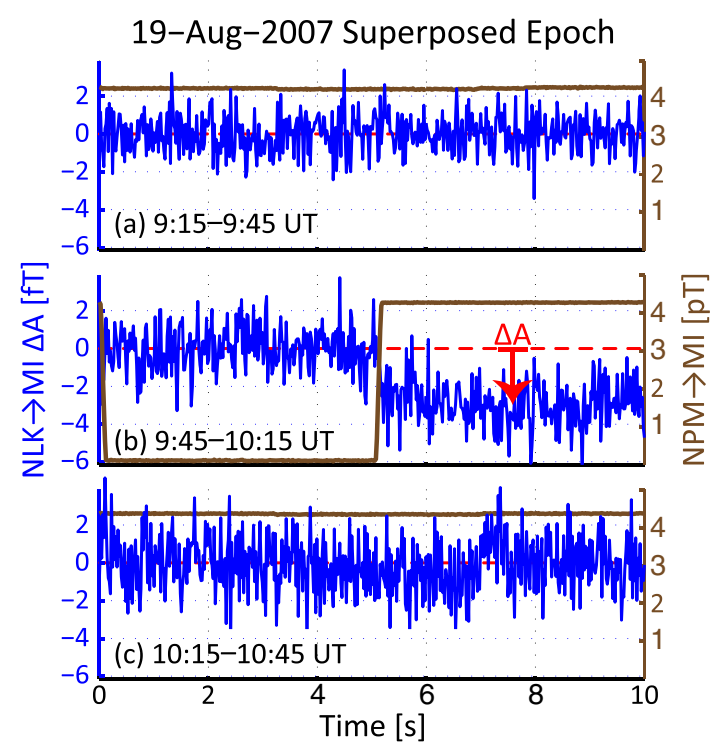

Figure 2. Experimental observations at MI on 19 August 2007. Plots present $10 \mathrm{~s}$ superposed epochs for (a) the $30 \mathrm{~min}$ prior to, (b) during, and (c) after the 9:45 to 10:15 UT NPM $5 \mathrm{~s}$ on/5 s off keying session. Received NPM signal amplitude is plotted in brown in pT as labeled on the right $y$ axis. Perturbation to the received NLK signal amplitude is plotted in blue in fT as labeled on the left $y$ axis. Figure $2 \mathrm{~b}$ clearly shows the NLK perturbation in tandem with the NPM on/off keying. This perturbation and keying are not present in the before (Figure 2a) or after (Figure 2c) plots.

of detectability for this set of NPM keying experiments. As such, we illustrate the theoretical NPM-induced electron precipitation region in Figure 1 only as a point of reference for discussion; Graf et al. [2011] has already shown that it does not influence the VLF measurements recorded in these experiments.

[12] A representative set of NPM keying session observations is presented in Figure 2 for the date of 19 August 2007. On this date, the NPM transmitter was keyed in a $5 \mathrm{~s}$ on/5 s off periodic format from 9:45 to 10:15 UT (11:45 P.M. to 12:15 A.M. local time), and the MI receiver recorded both the NPM and NLK signals. In each subplot of Figure 2, we plot the amplitude of the NPM-MI signal in brown, as labeled on the right-side $y$ axis, and we plot the change in amplitude of the NLK-MI signal in blue, as labeled on the left-side $y$ axis. Figure $2 \mathrm{a}$ is for the $30 \mathrm{~min}$ prior to the NPM keying session, Figure $2 \mathrm{~b}$ is for the 30 min during the keying session, and Figure $2 \mathrm{c}$ is for the $30 \mathrm{~min}$ after. Each plot shows the superposed epoch of each received signal, meaning we averaged over each $10 \mathrm{~s}$ block of data to improve the signal-to-noise ratio in detecting small periodic perturbations on the NLK-MI probe signal. The presence of impulsive noise due to sferics (the electromagnetic impulse from lightning discharges) common in narrowband VLF data would hinder this superposed epoch averaging, so we first replace any impulsive noise (defined as at least one standard deviation above the local $3 \mathrm{~s}$ median) of less than half a second in duration with the local mean of the data. In Figure $2 b$, the NLK-MI signal in blue is clearly perturbed instep with the NPM on/off keying. The NPM signal in brown is off for the first $5 \mathrm{~s}$ and on for the final $5 \mathrm{~s}$ of each $10 \mathrm{~s}$ period. When NPM switches from off to on, the NLKMI signal amplitude immediately decreases by $3 \mathrm{fT}$, which is approximately a $-0.25 \%$ change to its amplitude. A quick look at Figures $2 \mathrm{a}$ and $2 \mathrm{c}$ shows that during those neighboring times when NPM is not keying on/off (and is instead in continuous operation), the NLK-MI probe signal shows no such periodic perturbation. It is clear that the NPM on/off keying leads to the periodic perturbation of the NLK-MI probe signal.

[13] While we will not analyze signal phase in detail in this study, we provide the superposed epoch of the NLK-MI phase data in Figure 3 for the date of 19 August 2007. Just as in the amplitude data, the NLK-MI probe signal is clearly perturbed by the NPM on/off keying. Compared to probe signal amplitude, signal phase is typically more sensitive to small ionospheric disturbances. In addition, an amplitude perturbation does not necessarily coincide with a phase perturbation or vice versa [e.g., Barr et al., 1984]. Detection rates and perturbation analyses improve if both amplitude and phase data are available and reliable for analysis. Unfortunately, phase data is not easy to accurately extract and tends to be noisier, feature occasional phase jumps, and behave less consistently. In general, the NLK-MI phase data did not respond as well to this form of periodic perturbation analysis as did the NLK-MI amplitude data. Therefore, we will focus on probe signal amplitude perturbations for the bulk of the analysis.

[14] The previous plots presented NLK-MI probe signal perturbation analysis for a single $30 \mathrm{~min}$ NPM keying session. Over the course of the experiments, the NLK signal was recorded at MI for 1250 such keying sessions. Limiting analysis to keying sessions of the $5 \mathrm{~s}$ on/5 $\mathrm{s}$ off format and discarding any sessions for which either the NPM or NLK signals were weak or had signal dropouts, we are left with 930 keying sessions for analysis. For each of these 930 sessions, we perform the superposed epoch analysis as described above and compute the amplitude perturbation $\Delta A$

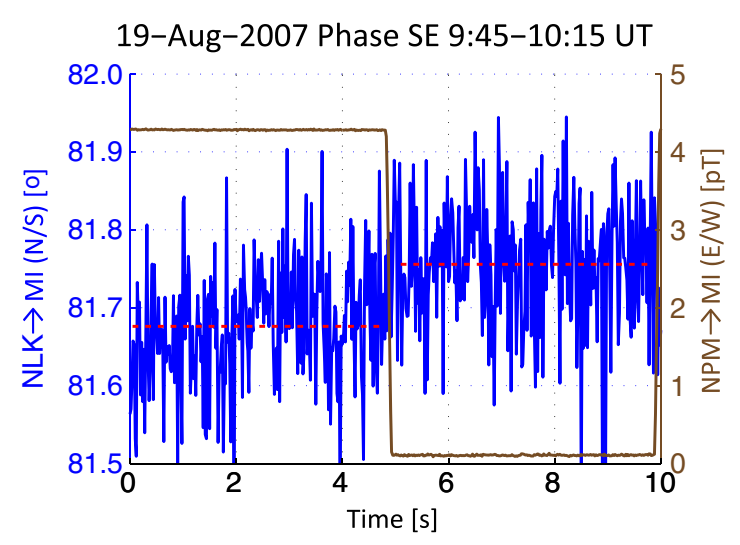

Figure 3. Experimental phase observations at MI on 19 August 2007. Plot presents $10 \mathrm{~s}$ superposed epoch for the $30 \mathrm{~min}$ during the $9: 45$ to $10: 15 \mathrm{UT}$ NPM $5 \mathrm{~s}$ on/5 $\mathrm{s}$ off keying session. Received NPM signal amplitude is plotted in brown as labeled on the right $y$ axis. The received NLK phase is plotted in blue as labeled on the left $y$ axis. 


\section{NLK $\rightarrow$ MI Probe Signal $\Delta \mathrm{A}$ Distribution}

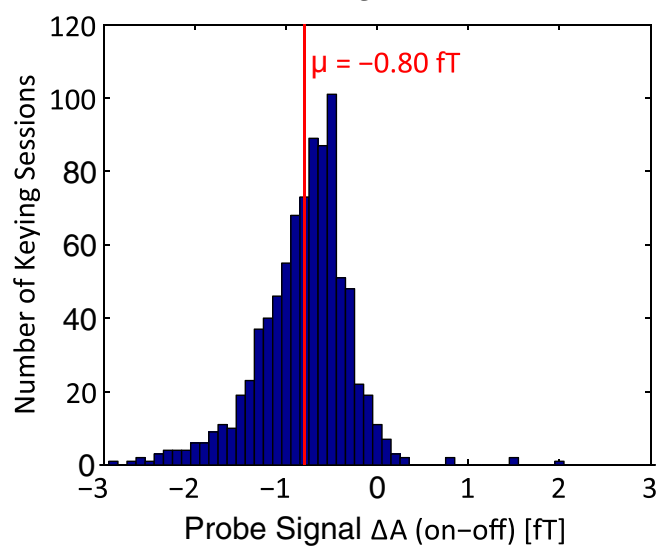

Figure 4. Distribution of perturbations to the NLK-MI probe signal for $93030 \mathrm{~min} 5 \mathrm{~s}$ on/5 s off nighttime NPM keying sessions. The average perturbation is $-0.80 \mathrm{fT}$, which is approximately a $-0.07 \%$ change.

of the NLK-MI probe signal. The value $\Delta A$ is computed during the NPM keying session as the average of the NLK-MI probe signal during the times when NPM is on minus the average of the NLK-MI signal during the times when NPM is off. The distribution of $\Delta A$ for these 930 keying sessions is presented in Figure 4. The result presented in Figure 2 was an extreme case at $-3 \mathrm{fT}$, but the NLK-MI probe signal is consistently perturbed by the NPM keying. Based on the typical noise levels of the received NLK-MI signal, most periodic perturbations greater than $0.5 \mathrm{fT}$ in magnitude would be detectable in the superposed epoch analysis, so the majority of the keying sessions produce a detectable perturbation to the NLK-MI signal. The average perturbation is $-0.8 \mathrm{fT}$. The average NLK-MI signal amplitude is $1.2 \mathrm{pT}$, so -0.8 fT represents a $-0.07 \%$ change. We also note that the perturbation is consistently negative, meaning the received NLK-MI signal is lower in amplitude when NPM is on.

[15] The superposed epoch analysis here is very much like that presented in Figure 2 of Graf et al. [2011] for the NPM keying session of 26 February 2008. As that study discussed in detail, the lack of onset delay between when the NPM transmitter switches on to when the perturbation begins to appear on the NLK-MI probe signal indicates that this perturbation could not have been due to NPM-induced electron precipitation. The observed onset delay is less than $50 \mathrm{~ms}$, while the time taken for NPM to initiate electron precipitation would be at least $200 \mathrm{~ms}$. Graf et al. [2011] also presented laboratory test results to show that these observations could not have been due to instrumental cross modulation. With both induced precipitation and instrumental cross modulation eliminated as possible causes for the observed NLK-MI probe signal perturbations, we investigate direct heating of the ionosphere by NPM as the possible link. We note first that ionospheric heating and cooling rates match the observed time signature of the perturbation. Heating occurs on the order of microseconds while cooling occurs in milliseconds, and given the time resolution of the narrowband data, both the perturbation onset delay and turnoff delay are less than $50 \mathrm{~ms}$. Thus, the perturbation time signature is consistent with ionospheric heating as the causative physical process. The computational modeling of the next section will serve to assess whether ionospheric heating by the NPM transmitter could realistically produce the magnitude of the observed probe signal perturbations.

\section{Computational Modeling}

[16] In this section we develop a large-scale computational model for ionospheric heating by the VLF transmitter NPM and the effect of this heating on the NLK-MI probe signal. The model consists of three steps: (1) computation of the wave electric field radiated by the NPM transmitter throughout the region of space, (2) computation of the increase in electron temperature generated by that wave electric field at each point, and (3) propagation of the NLK-MI probe signal through the heated Earth-ionosphere waveguide to estimate the amount it is perturbed by the NPM-induced ionospheric heating. This probe signal perturbation estimate is compared to that measured during the NPM transmitter keying experiments. Rodriguez et al. [1994] performed similar steps in their study of ionospheric heating by VLF transmitters, but their modeling only considered heating within $250 \mathrm{~km}$ radial distance of the transmitter. Here this distance is extended to $5000 \mathrm{~km}$.

[17] Note that we first compute the radiated wave electric field at each point in the domain and then compute the resultant increase in electron temperature separately as a post-processing step. This approach is not self-consistent due to the fact that a change in electron temperature would alter propagation of the heating wave itself, and thus, some form of feedback would occur. A change in electron temperature would also affect electron density [Rodriguez and Inan, 1994], which would in turn influence both wave propagation and heating behavior. While the decision to separate the wave propagation and ionospheric heating into separate steps without feedback is not ideal, the error associated with this lack of self-consistency should be very small, especially at great distances from the transmitter. This issue is discussed in more detail in section 4.3.

[18] Configurable media parameters input to the model are electron, nitrogen, and oxygen densities, ambient temperature, electron-neutral collision frequency, and background geomagnetic field. Ambient profiles used in this study are presented in Figure 5. This electron density profile was used by Taranenko et al. [1992] and as profile II of Inan et al. [1992]. It is based on data from the International Reference Ionosphere model [Rawer et al., 1978; Bilitza and Reinisch, 2008] and represents a typical nighttime ionosphere electron density profile to be used throughout the region for ambient conditions. The neutral particle densities and ambient temperature profiles are from the NRLMSISE00 model [Picone et al., 2002] for local midnight, and collision frequency is based on Swamy [1992]. Each of these ionospheric profiles varies with altitude but do not vary latitude or longitude within our model. In other words, we use each of the profiles plotted in Figure 5 throughout the domain for ambient conditions. We do, however, vary the background geomagnetic field throughout the region by using values from the IGRF10 model [Macmillan and Maus, 2005] for each point in latitude, longitude, and altitude. 
(a) Particle Densities ( $N$ )

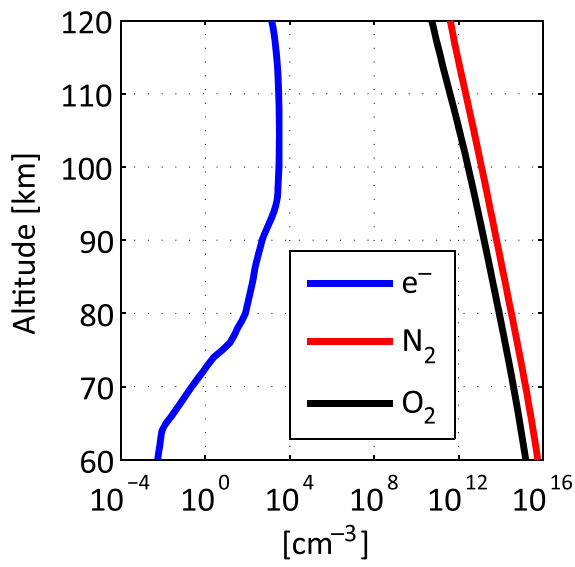

(b) Temperature $\left(T_{0}\right)$

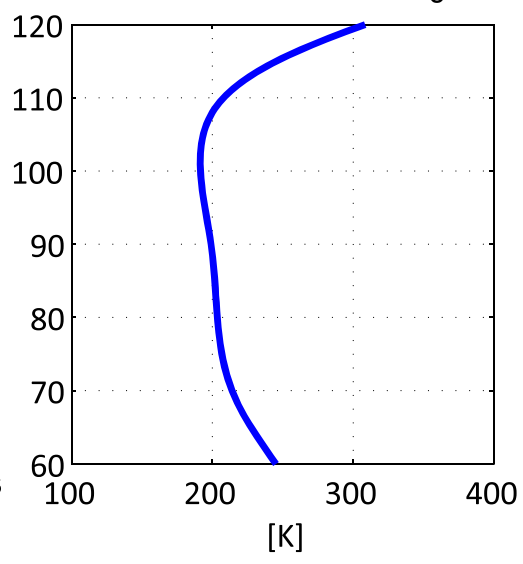

(c) Electron-Neutral Collision Frequency $(v)$

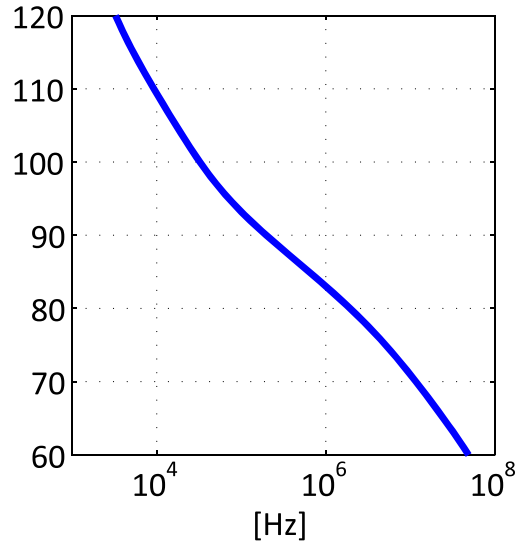

Figure 5. Ambient nighttime ionosphere profiles to be used throughout the computational model. Ambient temperature and neutral densities are from the NRLMSISE-00 [Picone et al., 2002]. Electron density is from profile II of Inan et al. [1992], and collision frequency is based on Swamy [1992]. Geomagnetic field (not shown here) is from IGRF10 [Macmillan and Maus, 2005].

\subsection{NPM-Radiated Fields}

[19] To compute the ionospheric heating generated by the VLF transmitter NPM at great distances, we first compute the radiated wave electric field at each point in the domain. Computing the wave electric field throughout the entire region of $5000 \mathrm{~km}$ radius is problematic because it is simply too large for a full 3-D model considering available computational resources. We navigate this issue by computing the wave propagation individually along many 2-D paths in the Earth-ionosphere waveguide extending radially from the NPM transmitter. We then combine these paths to fill the 3-D space. We compute wave propagation along these 2-D paths with a segmented long path (SLP) arrangement of the discontinuous Galerkin (DG) method [Foust et al., 2011]. In addition to handling basic wave propagation in the Earth-ionosphere waveguide, this model also accurately accounts for spreading of the wave energy, curvature of the Earth, inhomogeneous background magnetic field, and ground conductivity, and can accurately compute wave electric field penetrating up into the ionosphere. The model is accurate for great distances from the source, but due to the source implementation as a ring offset from the pole in the simulation space, it does not accurately represent the fields near the transmitter. For this reason, we only use data from this DG model for distances greater than $500 \mathrm{~km}$ from the VLF transmitter NPM, and we use a different model for closer distances. For distances within $500 \mathrm{~km}$ of NPM, we use the Stanford Full Wave Method (FWM) [Lehtinen and Inan, 2008, 2009]. The FWM model assumes horizontally stratified media, so it cannot currently account for Earth curvature or horizontally varying geomagnetic field or ground conductivities. For within $500 \mathrm{~km}$ of the source, however, horizontal stratification is a good approximation and we can very accurately compute radiated wave electric field. This FWM model has been validated for VLF propagation into and through the ionosphere [Cohen et al., 2012; Graf et al., 2013] and has also been used for propagation within the Earth-ionosphere waveguide [Lehtinen et al., 2010].
[20] The vertical component of the wave electric field radiated by the NPM transmitter for within $500 \mathrm{~km}$ lateral distance is presented in Figures 6a and 6b. The field values were computed with the FWM model. Figure 6a shows the fields for a vertical slice running from South to North. A null is directly above the transmitter located at $0 \mathrm{~km}$, and most of the wave energy is reflected by the lower ionosphere. We also see North/South asymmetry due to geomagnetic field orientation, with greater penetration into the ionosphere toward the South where propagation is more closely along the background geomagnetic field as opposed to across it. Figure $6 \mathrm{~b}$ shows the fields for a horizontal plane taken at 80 $\mathrm{km}$ altitude above the NPM transmitter. The source is located at the origin in the center of the image. A null is directly above the transmitter, and concentric rings form in the radiation pattern due to the mode structure established by wave propagating in the Earth-ionosphere waveguide below.

[21] The electric field radiated by the NPM transmitter for distances greater than $500 \mathrm{~km}$ is presented in Figures 7 and $8 \mathrm{a}$. These values are computed by using the DG model. Figure 7 presents the vertical component of the wave electric field for along a single 2-D path extending radially from the NPM transmitter at a bearing angle of $300^{\circ}$. The wave propagates to great distances in the Earth-ionosphere waveguide, with the limited attenuation that does occur being due to a combination of collisional losses, leakage out of the waveguide, and radial spreading of the wave energy. We note that at these greater distances from the transmitter, the wave amplitude attenuates at as little as 2 to $3 \mathrm{~dB} / \mathrm{Mm}$. This agrees with Crary [1961] and indicates that significant field strengths exist even at several thousand kilometers distance from the transmitter. This 2-D path of Figure 7 results from a single run of the DG code. We combine 36 of these 2-D runs, at each $10^{\circ}$ step in bearing angle, to compute the electric field radiated by NPM throughout the 3-D region. This approach gives relatively poor azimuthal resolution at great distances but still clearly illustrates the structure of the radiated fields, and the resolution is sufficient for the present 

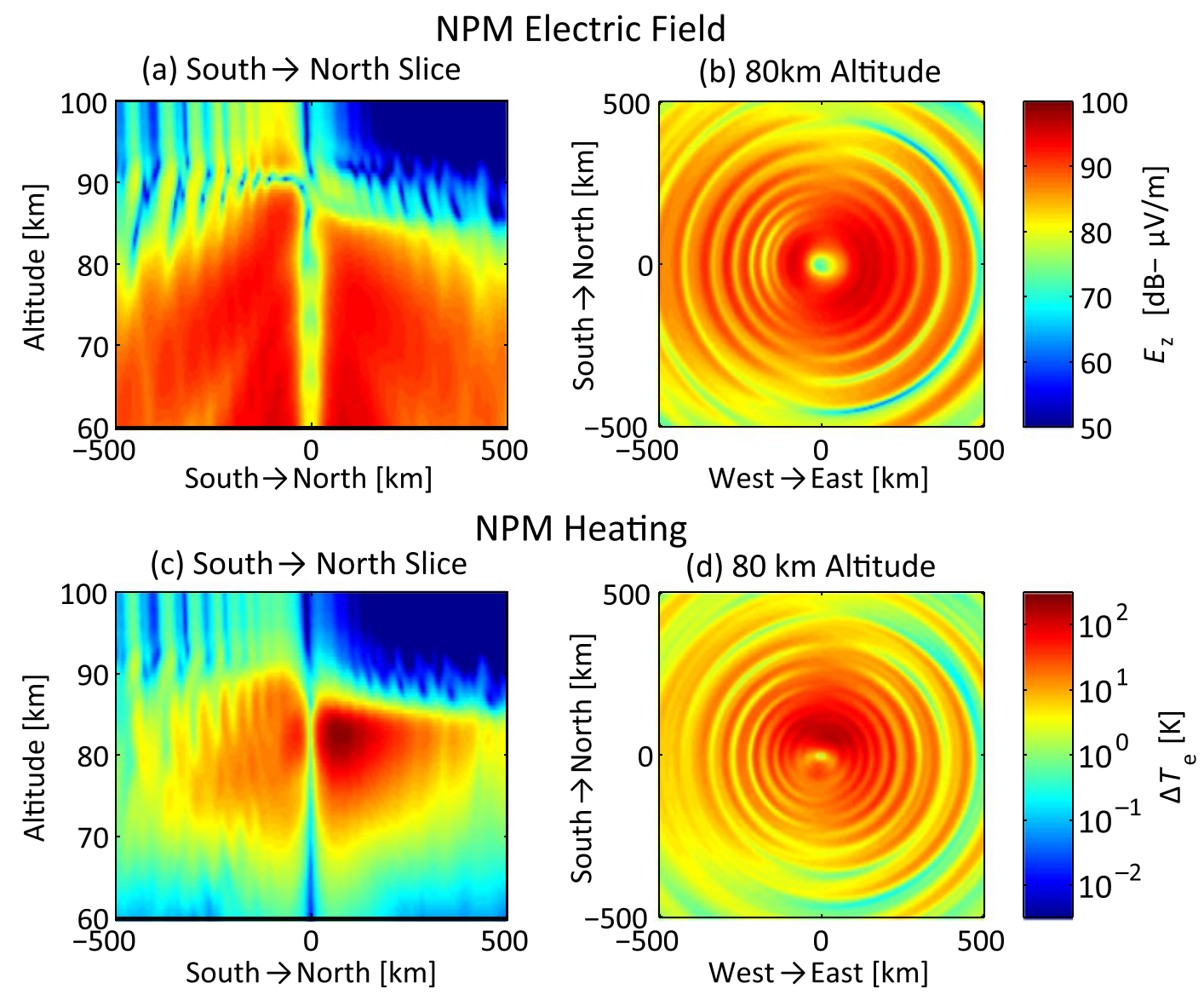

Figure 6. ( $a, b)$ Vertical component of electric field radiated by NPM, as computed by the Stanford FWM model for within $500 \mathrm{~km}$ radius, for vertical slice running from South to North (Figure 6a) and horizontal plane at $80 \mathrm{~km}$ altitude (Figure 6b). (c, d) Ionospheric heating induced by the NPM transmitter within $500 \mathrm{~km}$ radial distance, as computed using electric field values computed by the Stanford FWM model. The spatial domain for Figure 6c corresponds directly to that of Figure 6a, and Figure 6d corresponds to Figure $6 b$.

application. The electric field values for a horizontal plane at $80 \mathrm{~km}$ altitude are presented in Figure $8 \mathrm{a}$, where the values are plotted onto a map for reference. NPM is located in Hawaii in the center of the map, and the West coast of North America is visible in the upper right.

[22] While the wave amplitudes computed by the FWM model are accurate for a specified source power, the DG model assumes a source of arbitrary unit amplitude and the results require scaling. We compute a single scaling factor for the DG model results for NPM by performing a least squares fit to a combination of recorded field values at VLF receiver sites and field values estimated by the FWM model. Three VLF receiver sites were available for comparison: Midway Atoll (MI; $\left.28.21^{\circ} \mathrm{N}, 177.38^{\circ} \mathrm{W}\right)$, Valdez, Alaska (VZ; $\left.61.06^{\circ} \mathrm{N}, 146.02^{\circ} \mathrm{W}\right)$, and Juneau, Alaska (JU; $\left.58.59^{\circ} \mathrm{N}, 134.90^{\circ} \mathrm{W}\right)$. All three recorded the NPM signal strength at their location throughout the keying experiments. We compute the average NPM field value recorded at each site during the times when NPM was switched on during the keying sessions and compare this to the field value predicted at each location by the DG model. Since this provides just three distant points for comparison, we include four additional points from the FWM model: one in each of the four cardinal directions at approximately $500 \mathrm{~km}$ radial distance where the two models merge. Model results are analyzed in the vicinity at each of these points to ensure that local extrema in field amplitude do not disrupt the scaling. After computing the universal DG model scaling factor based on these seven points of comparison, all field values computed by the DG model are scaled accordingly. A comparison of the scaled DG model results to the seven reference measurements is provided in Figure 9. All of the values are in close agreement, with no comparison showing more than $20 \%$ discrepancy.

\subsection{Ionospheric Heating}

[23] With the electric field computed at each point extending to $5000 \mathrm{~km}$ radial distance from the NPM transmitter, we now solve the energy balance equation for electron heating. When balancing electron heating, $U$, with electron cooling, $L_{e}$, it follows that

$$
\frac{3}{2} k_{\mathrm{B}} N_{e} \frac{\mathrm{d} T_{e}}{\mathrm{~d} t}=U-L_{e}
$$

where $k_{\mathrm{B}}$ is Boltzmann's constant, and $N_{e}$ and $T_{e}$ are electron density and temperature, respectively. 


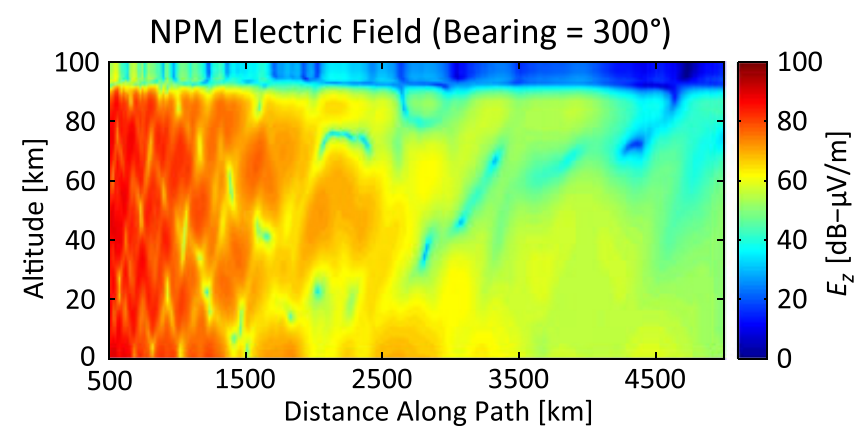

Figure 7. Vertical component of electric field radiated by NPM, as computed by the DG code for distances 500 to $5000 \mathrm{~km}$. Plot shown is for a 2-D path extending radially from the NPM transmitter at a bearing of $300^{\circ}$.

[24] Electron heating, $U$, due to the wave electric field accelerating the lightweight electrons, can be computed most directly from the expression for Joule heating:

$$
U=\frac{1}{2} \operatorname{Re}\left(\vec{J}^{*} \cdot \vec{E}\right),
$$

where $\vec{E}$ is the wave electric field vector and $\vec{J}=\overline{\bar{\sigma}} \cdot \vec{E}$ is current density. The conductivity matrix $\overline{\bar{\sigma}}$ is a function of electron density, collision frequency, geomagnetic field, and wave frequency [Bittencourt, 2005, p. 247]. Combining the ambient media parameters with the computed wave electric field allows for the direct computation of electron heating $U$. We compute $U$ at each point as a post-processing step after completing the wave propagation model.

[25] Electron cooling, $L_{e}$, accounts for elastic, rotational, and vibrational transfer of energy to $\mathrm{N}_{2}$ and $\mathrm{O}_{2}$ and is a function of electron density, neutral densities, ambient temperature, and electron temperature. The following expression for $L_{e}$ was derived through a combination of empirical and theoretical analyses by Mentzoni and Row [1963] and Dalgarno et al. [1968] (rotational), Banks [1966] (elastic), Stubbe and Varnum [1972] (vibrational), and Schunk and Nagy [1978] (total) and was used previously by Rodriguez et al. [1994]:

$$
\begin{aligned}
L_{e}= & \left(L_{\mathrm{rot}}\left(\mathrm{e}^{-}, \mathrm{N}_{2}\right)+L_{\mathrm{elast}}\left(\mathrm{e}^{-}, \mathrm{N}_{2}\right)+L_{\mathrm{vib}}\left(\mathrm{e}^{-}, \mathrm{N}_{2}\right)\right) \ldots \\
& +\left(L_{\mathrm{rot}}\left(\mathrm{e}^{-}, \mathrm{O}_{2}\right)+L_{\mathrm{elast}}\left(\mathrm{e}^{-}, \mathrm{O}_{2}\right)+L_{\mathrm{vib}}\left(\mathrm{e}^{-}, \mathrm{O}_{2}\right)\right) \\
L_{\mathrm{rot}}\left(\mathrm{e}^{-}, \mathrm{N}_{2}\right)= & 4.65 \times 10^{-39} N_{e} N_{\mathrm{N}_{2}} \frac{T_{e}-T_{0}}{\sqrt{T_{e}}} \\
L_{\mathrm{rot}}\left(\mathrm{e}^{-}, \mathrm{O}_{2}\right)= & 1.11 \times 10^{-38} N_{e} N_{\mathrm{O}_{2}} \frac{T_{e}-T_{0}}{\sqrt{T_{e}}} \\
L_{\mathrm{elast}}\left(\mathrm{e}^{-}, \mathrm{N}_{2}\right)= & 1.89 \times 10^{-44} N_{e} N_{\mathrm{N}_{2}} \\
L_{\mathrm{elast}}\left(\mathrm{e}^{-}, \mathrm{O}_{2}\right)= & \left.1.29 \times 10^{-43} N_{e} N_{\mathrm{O}_{2}}\right) T_{e}\left(T_{e}-T_{0}\right) \\
& \cdot\left(1+3.6 \times 10^{-2} \sqrt{T_{e}}\right) \sqrt{T_{e}}\left(T_{e}-T_{0}\right) \\
L_{\mathrm{vib}}\left(\mathrm{e}^{-}, \mathrm{N}_{2}\right)= & 4.79 \times 10^{-37} N_{e} N_{\mathrm{N}_{2}} \exp \left[f_{\mathrm{N}_{2}} \frac{\left(T_{e}-2000\right)}{2000 T_{e}}\right] \\
& \cdot\left(1-\exp \left[-g \frac{T_{e}-T_{0}}{T_{e} T_{0}}\right]\right) \\
L_{\mathrm{vib}}\left(\mathrm{e}^{-}, \mathrm{O}_{2}\right)= & 8.32 \times 10^{-38} N_{e} N_{\mathrm{O}_{2}} \exp \left[f_{\mathrm{O}_{2}} \frac{\left(T_{e}-700\right)}{700 T_{e}}\right] \\
& \cdot\left(1-\exp \left[-2700 \frac{T_{e}-T_{0}}{T_{e} T_{0}}\right]\right) \\
& \left(1.06 \times 10^{4}\right)+\left(7.51 \times 10^{3}\right) \\
& \cdot \tanh \left(0.0011\left(T_{e}-1800\right)\right) \\
f_{\mathrm{N}_{2}}= & 3300-839 \sin \left(0.000191\left(T_{e}-2700\right)\right) \\
g= & 3300+1.233\left(T_{e}-1000\right)-\left(2.056 \times 10^{-4}\right) \\
& \cdot\left(T_{e}-1000\right)\left(T_{e}-4000\right)
\end{aligned}
$$

Typical values for the cooling rate terms as applicable to VLF heating of the lower nighttime ionosphere are shown in Figure 10. For this regime, electron cooling is dominated by the rotational transfer of energy. Vibrational energy transfer becomes dominant at much higher electron temperatures $\left(\Delta T_{e}>1000 \mathrm{~K}\right)$, but ionospheric heating by ground-based VLF transmitters is not expected to exceed $\Delta T_{e} \approx 500 \mathrm{~K}$.



(b) NPM Heating

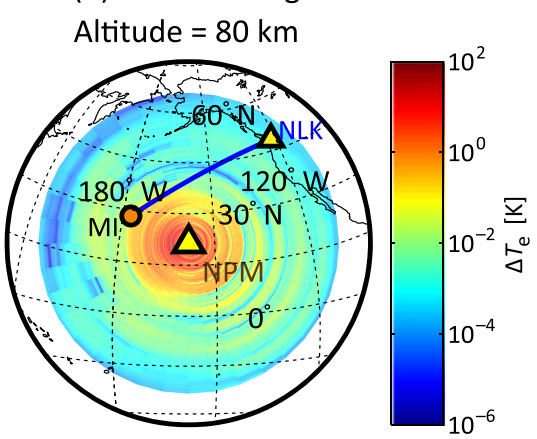

Figure 8. (a) Vertical component of electric field radiated by NPM, as computed by the DG code for radial distances up to $5000 \mathrm{~km}$. Plot shown is for $80 \mathrm{~km}$ altitude. Results generated by combining 36 2-D radial paths; one of which is shown in Figure 7. (b) Map of ionospheric heating at $80 \mathrm{~km}$ altitude induced by the NPM transmitter. Location of the NPM transmitter is marked in the center of each image. Great circle path of the NLK to MI probe signal is drawn in blue. 


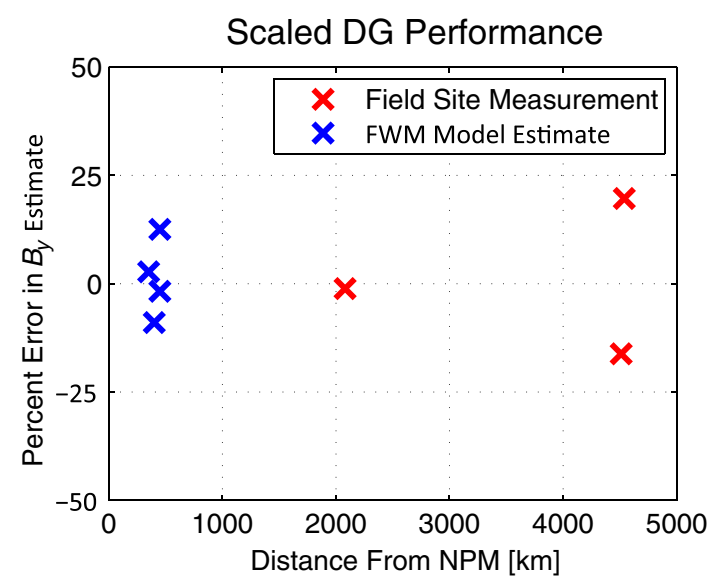

Figure 9. Performance of the scaled DG model for estimating fields radiated by the NPM transmitter. Comparisons are made to sample points from the FWM model in the four cardinal directions (blue) and to long-term averaged measurements from three available VLF receiver sites (red). Field values estimated by the scaled DG model show less than $20 \%$ discrepancy at each point of comparison.

[26] Following the computation of wave electric field $\vec{E}$ and setting the ambient media parameters, the only remaining unknown in equation (1) is the electron temperature $T_{e}$. Solving equation (1) in this application is greatly simplified because we need only consider the steady state solution. Since the heating and cooling rates are both on the order of $1 \mathrm{~ms}$ or less and the data sampling rate in the experiments is $20 \mathrm{~ms}$, the heating process can be treated as instantaneous. Setting $\mathrm{d} T_{e} / \mathrm{d} t=0$, we need only numerically solve the resultant equation $U=L_{e}$ for $T_{e}$. Performing this task at each point in the model produces the electron-heating values presented in Figure 8b. In these computations, we use FWM electric field values for within $500 \mathrm{~km}$ radial distance and DG electric field values for 500 to $5000 \mathrm{~km}$ radial distance. For $80 \mathrm{~km}$ altitude, there is on the order of $100 \mathrm{~K}$ electron temperature increase near NPM (approximately a $50 \%$ change), $1 \mathrm{~K}$ increase at $1000 \mathrm{~km}$ radial distance, and 0.01 to $0.1 \mathrm{~K}$ increase at $3000 \mathrm{~km}$ radial distance. A closer look at the heating near NPM is provided in Figures $6 \mathrm{c}$ and $6 \mathrm{~d}$, where only the electric field values computed by the FWM model are required. These results for ionospheric heating near NPM compare favorably to those of Rodriguez et al. [1994], both in the general structure and intensity of the heating region.

[27] The region of space plotted in Figure $6 \mathrm{c}$ and $6 \mathrm{~d}$ compares directly to that of Figures $6 a$ and $6 b$, and Figure 8a compares directly to Figure $8 \mathrm{~b}$. Electron-heating structure roughly matches that of the wave electric field amplitude, and discrepancies arise primarily due to the anisotropic conductivity matrix. For example, in Figures $6 \mathrm{a}$ and $6 \mathrm{~b}$ there is greater penetration of the wave electric into the ionosphere to the South of NPM, but in Figures $6 \mathrm{c}$ and $6 \mathrm{~d}$ there is greater heating at $80 \mathrm{~km}$ altitude to the North. Physically, this is because propagation to the North across the geomagnetic field means the wave electric field is more closely aligned with that geomagnetic field, thus taking advantage of increased electron mobility to produce greater current densities and greater electron heating.

\subsection{Probe Signal Perturbation}

[28] Now that we have estimated the ionospheric heating induced by the keyed NPM transmitter, we assess the impact of this heating on the subionospherically propagating NLKMI probe signal. To estimate the probe signal perturbation, we propagate the NLK probe signal through the Earthionosphere waveguide to the MI receiver under both ambient and disturbed ionospheric conditions. The great circle path of the NLK-MI probe signal is traced in blue in Figure $8 \mathrm{~b}$. This pathway is approximately $5200 \mathrm{~km}$ in length and is $1750 \mathrm{~km}$ from the NPM transmitter at its point of closest approach. Since this full 3-D region is too large to model as a single scattering problem, we focus on propagation and scattering along the 2-D NLK-MI pathway. This approach ignores the effects of off-path scattering, a decision which we will discuss and justify in section 4.2.

[29] To estimate the probe signal propagation under ambient conditions we employ the same ambient ionospheric profiles and geomagnetic field models used in the previous modeling steps. For propagation under disturbed conditions, we extract the change in electron temperature along the NLK-MI pathway from the ionospheric heating model results. Temperature change is proportional to collision frequency change for these low-energy electrons [Budden, 1985, p. 58], and collision frequency affects the conductivity matrix which dictates wave propagation and scattering [Bittencourt, 2005, p. 247]. The change in electron-neutral collision frequency along the NLK-MI pathway is presented in Figure 11. Changes to the collision frequency are minimal (less than $0.01 \%$ ), close to the NLK transmitter along this path where it is still 3000 to $5000 \mathrm{~km}$ from the heating NPM transmitter, but collision frequency

\section{Typical Cooling Rates at $85 \mathrm{~km}$ Altitude, Nighttime}

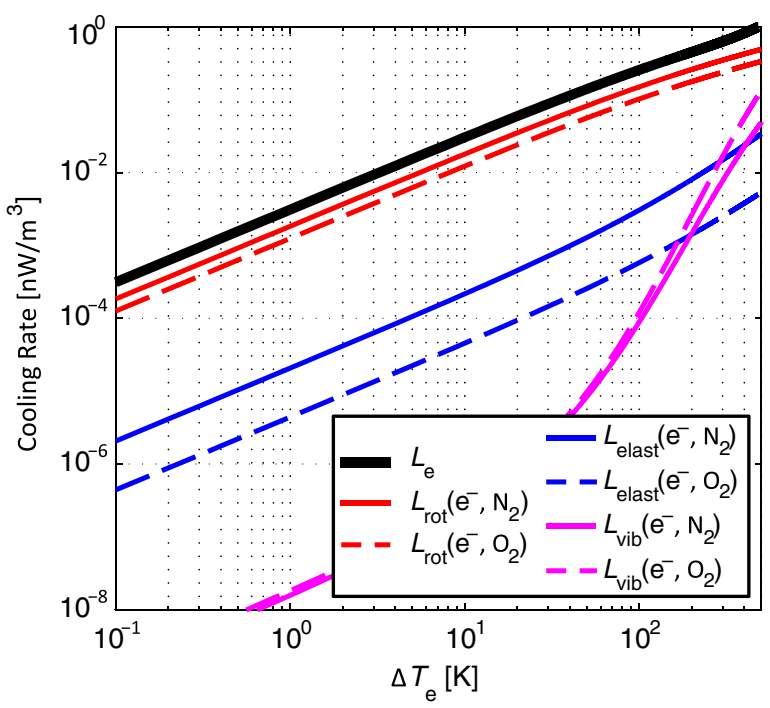

Figure 10. Typical values of the cooling rate terms for $85 \mathrm{~km}$ altitude in the nighttime ionosphere. The range of $\Delta T_{e}$ shown is applicable for ionospheric heating by a ground-based VLF transmitter. 
changes as large as $1 \%$ occur further along the pathway where it approaches within $2000 \mathrm{~km}$ of NPM.

[30] For the 2-D probe signal propagation and perturbation analysis, we use the SLP arrangement of the finite-difference frequency-domain (FDFD) code developed by Chevalier and Inan [2006] and Chevalier et al. [2008]. This code has been used in multiple previous studies [e.g., Chevalier et al., 2007; Marshall et al., 2008; Marshall and Inan, 2010] to estimate the perturbation to a subionospherically propagating VLF probe signal induced by an ionospheric disturbance. Using this FDFD model, we propagate the NLK to MI probe signal under both ambient and disturbed conditions. The results are presented in Figure 12. Figure $12 \mathrm{a}$ shows the propagation under ambient conditions, with the NLK transmitter located at $0 \mathrm{~km}$, and the MI receiver near the right edge at $5200 \mathrm{~km}$. Since the change to this probe signal under disturbed ionospheric conditions is very small, we plot the perturbation to the probe signal in Figure 12b. We compute the perturbation as (disturbed)-(ambient), with red indicating a positive perturbation, blue indicating a negative perturbation, and white indicating no change. The largest probe signal perturbations occur further in the waveguide, once the wave encounters the region of more intense heating seen further in the waveguide in Figure 11. Since the VLF receiver MI is located on the ground, we extract the probe signal perturbation estimated along the ground and plot this in Figure 12c. Assuming these typical ionospheric conditions, this model estimates an observation of $\Delta A \approx-0.1$ to $+0.1 \mathrm{fT}$ near MI. For comparison, we mark the average MI observation in pink: $\Delta A \approx$ $-0.8 \mathrm{fT}$ at a distance of $5200 \mathrm{~km}$ from the NLK transmitter.

[31] Throughout all modeling steps, we have assumed the "typical" ambient ionospheric electron density profile of Taranenko et al. [1992] and Inan et al. [1992]. For comparison, we also executed the entire model (NPM transmission, ionospheric heating, and probe signal propagation) with a "tenuous" ionosphere. Based on statistical bounds from in situ electron density values measured in several rocket studies [Tao et al., 2010] and following the lead of Cohen et al. [2012] and Graf et al. [2013], we divide the "typical" electron density profile by a factor of 5 to define the "tenuous" nighttime ionosphere. While the intermediate modeling results are not presented here, we include the probe signal perturbation estimated along the ground for a tenuous ionosphere in Figure 12c. The model now predicts an observation of $\Delta A \approx-1.2 \mathrm{fT}$ at MI.

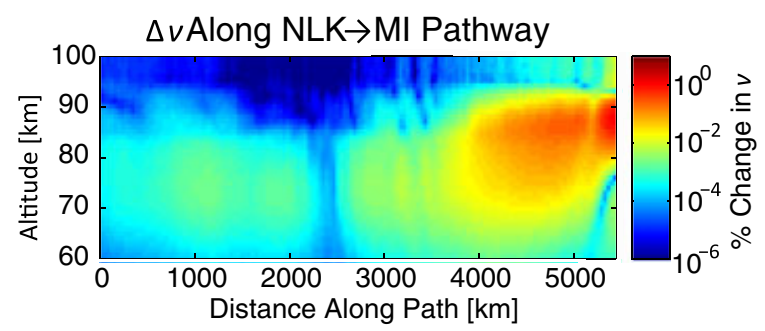

Figure 11. NPM-induced ionospheric heating along the NLK-MI probe signal pathway as extracted from results of the large-scale heating model. Ionospheric profiles and heating are defined above $60 \mathrm{~km}$ altitude; 0 to $60 \mathrm{~km}$ altitude is treated as free space.
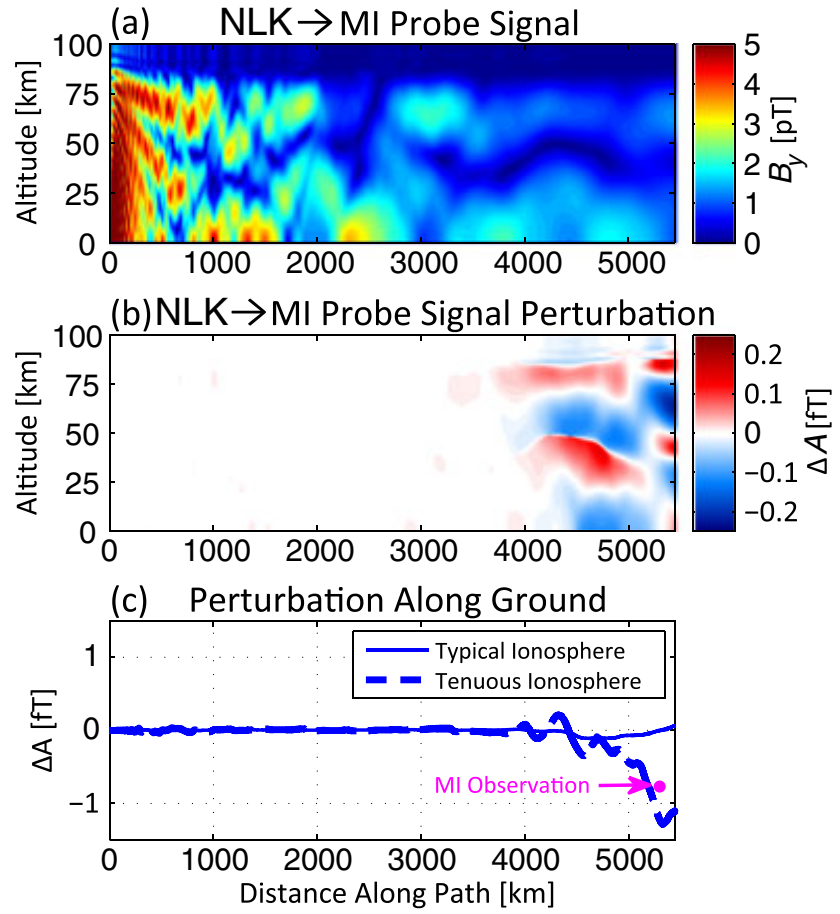

Figure 12. Perturbation to the NLK-MI probe signal due to NPM-induced extended lateral heating of the nighttime ionosphere. (a) Propagation of the NLK probe signal through the Earth-ionosphere waveguide to the MI receiver. (b) Perturbation to the NLK probe signal due to the NPM-induced ionospheric heating presented in Figure 11 for a typical ionosphere. (c) Perturbation to the NLK probe along the ground, as would be measured by a VLF receiver detecting the local transverse magnetic field component, which includes results for both a typical ionosphere and a tenuous ionosphere, together with the typical MI experimental observation marked in pink.

\section{Discussion}

[32] During the NPM keying experiments, we consistently detected a perturbation on the NLK probe signal received at $\mathrm{MI}$. The average perturbation was $-0.8 \mathrm{fT}$. Computational modeling of ionospheric heating and probe signal propagation estimated a perturbation of approximately \pm 0.1 fT on this probe signal for a typical ionosphere, and $-1.2 \mathrm{fT}$ for tenuous ionosphere. So within the error due to input variability, the model results agree with the experimental observations in both sign and magnitude. This suggests that extended lateral heating of the nighttime ionosphere by the keyed NPM transmitter can realistically account for the observed NLK-MI probe signal perturbations. The fact that the detected perturbation is preferentially negative in both observation and model matches general intuition, but we would not expect this convenient result to translate to all scattering geometries. An increase in electron-neutral collision frequency due to ionospheric heating not only increases the ionospheric reflection height of the VLF probe signal but also increases the collision frequency at that reflection height. The end result is greater attenuation of the probe signal and an overall decrease in amplitude. 
However, despite the overall decrease in probe signal amplitude, the perturbation analysis in Figure 12b shows that there will still be patches where $\Delta A$ is positive and patches where $\Delta A$ is negative. This is due to the impact of the ionospheric disturbance on the mode structure of the VLF probe signal propagating in the Earth-ionosphere waveguide. A slightly different scattering geometry could put the receiver in a location where the detected perturbation was positive or even zero.

\subsection{Ionospheric and Geomagnetic Variability}

[33] While the use of typical ambient media profiles throughout the region underestimated the observed perturbation by a factor of 8 in the model, the analysis of a tenuous ionosphere showed that this discrepancy was well within the general variability of the model inputs. A tenuous ionosphere produces multiple effects which increase the probe signal perturbation: less attenuation of the NPM signal with distance, greater penetration of the NPM signal into the ionosphere, increased ionospheric heating, and greater penetration of the NLK probe signal into the ionosphere to interact with greater ionospheric disturbances. In comparison to a uniformly typical or dense ionosphere, the existence even of patches of tenuous ionosphere along the NLK-MI pathway could greatly increase the observed probe signal perturbation because heating and scattering in this tenuous region would likely dominate over the heating and scattering from nearby regions of denser ionospheric electron density. We compared the model results for typical and tenuous ionospheric electron density profiles primarily as a representative assessment of model error due to variability of the input media profiles. Ionospheric electron density is the most variable of the input media profiles, but changes to the ambient temperature, collision frequency, neutral densities, geomagnetic field, and ground conductivities would also contribute both to model error and to the variability of the observed probe signal perturbations.

[34] In addition to the general day-to-day and placeto-place variability of ionospheric electron density, the ionosphere also experiences a known seasonal trend due to variation in local solar flux over the course of a year. In Figure 13, we analyze the experimental observations for the presence of a seasonal trend. In this figure, each red dot represents the probe signal perturbation computed for a 30 min NPM keying session on that day. The black curve marks the running mean and standard deviation of those red points. As a proxy for the seasonal trend of ionospheric electron density, we plot in blue the electron density over the course of the year from the International Reference Ionosphere model [Bilitza and Reinisch, 2008] for a point at $85 \mathrm{~km}$ altitude along the NLK-MI path near the NPM heating region in the year 2007 at local midnight. For this region of the world, ionospheric electron density peaks in the late spring and early summer months. While there is significant day-to-day spread in the observed probe signal perturbation (likely due in large part to day-to-day ionospheric variability), there also appears to be a seasonal trend: the detected probe signal perturbation is lowest during the months when ionospheric electron density is highest. This agrees with our general intuition and the computational modeling results: when the ionosphere is denser, the ionospheric heating and probe signal perturbation are both weaker.

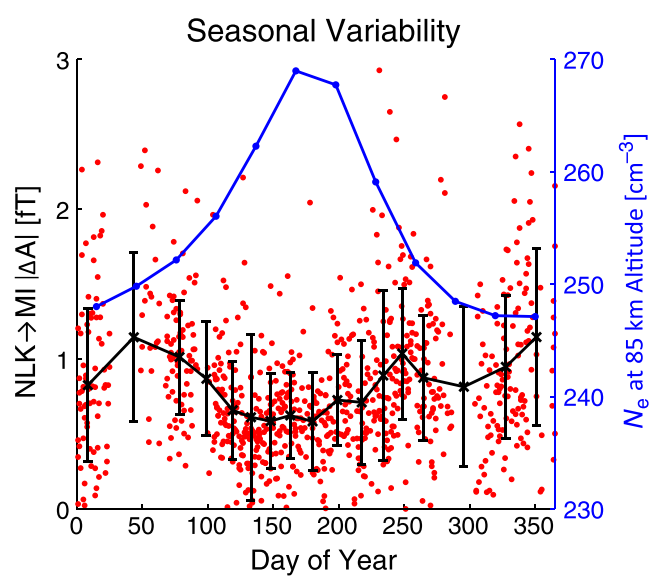

Figure 13. Seasonal variability of the NLK-MI probe signal perturbation together with seasonal variability of ionospheric electron density. Each red point marks the probe signal perturbation computed for a $30 \mathrm{~min}$ NPM keying session on that day. The black curve marks their running mean and standard deviation. The blue curve marks the electron density at $85 \mathrm{~km}$ altitude over the course of the year as estimated by the IRI-2007 model [Bilitza and Reinisch, 2008] for a point along the NLK-MI path in the year 2007 at local midnight.

[35] We also compare the amplitude of the observed probe signal perturbations to geomagnetic activity and present the correlation of $|\Delta A|$ with $A p$ index in Figure 14. This analysis shows that there is no correlation between geomagnetic activity and the observations. This is to be expected if ionospheric heating is the linking physical phenomenon. Beyond the impact of increased geomagnetic activity on ionospheric electron density (which should only have a minor effect on these midlatitude observations), there should be no strong link between geomagnetic activity and ionospheric heating by a ground-based VLF transmitter. In addition, this lack of correlation with $A p$ index lends further support to the case made by Graf et al. [2011] that the observed probe signal perturbations could not have been due to transmitter-induced precipitation of electron radiation. Geomagnetic activity is connected to the flux levels and pitch angle distributions of trapped electrons in the Earth's radiation belts. Strong geomagnetic activity can "prime" the trapped energetic electron population, creating a "top hat" pitch angle distribution where many particles are close to being scattered into the bounce loss cone [Lyons and Williams, 1975]. Once the pitch angle distribution is primed in this manner, a resonant VLF wave can readily scatter more trapped energetic electrons into the bounce loss cone, potentially producing far greater electron precipitation effects. Leyser et al. [1984] and Peter and Inan [2004] assessed this effect for the observation of lightning-induced electron precipitation (LEP) and suggested that there is a relationship between geomagnetic activity and the conditions conducive to the occurrence of detectable LEP events. We would expect a similar relationship between geomagnetic activity and the detection of transmitter-induced electron precipitation. Since the observations show no correlation with geomagnetic activity, it is unlikely that they would be due to induced electron precipitation. 


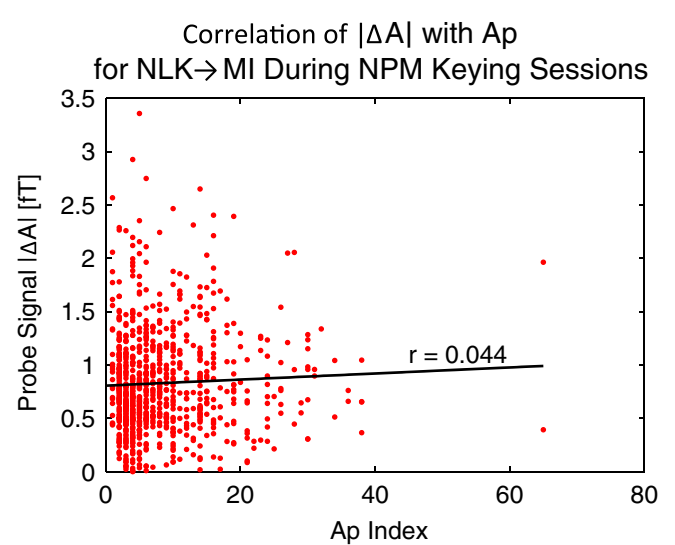

Figure 14. Correlation of the NLK-MI probe signal perturbation with geomagnetic activity index $A p$. No correlation is found.

\subsection{Along-Path Versus Off-Path Scattering}

[36] While assembling the computational model, we made several decisions in order to model the heating and scattering with sufficient accuracy over the very large distances involved. One such decision that warrants further analysis and justification is the choice to limit the probe signal perturbation to strictly a 2-D along-path scattering analysis. In reality, studying the NLK-MI probe signal during the NPM keying experiments presents a large-scale 3-D scattering problem within the spherical shell Earth-ionosphere waveguide. A strong ionospheric disturbance exists some distance $(\sim 1500 \mathrm{~km})$ away from the probe signal pathway, and weak, diffuse ionospheric disturbance extends to great distances $(\sim 2000 \mathrm{~km})$ covering the probe signal pathway itself. The NLK probe signal will scatter from the distant off-path disturbance, the extended along-path disturbances, and everything in between. In general, for different scattering geometries and ionospheric disturbances, there will be situations where the off-path scattering dominates and situations where the along-path scattering dominates. For practical purposes, we assess the along-path scattering and the off-path scattering separately for our situation and then focus the modeling efforts on the dominant effect if we conclude that the other effect is likely inconsequential. For the NPM-NLK-MI scattering geometry, the NLK-MI great circle path is never closer than $1750 \mathrm{~km}$ to NPM, and the required forward-scattering angle to reach MI from NPM for the NLK probe signal is $77^{\circ}$. Wide-angle scattering would be required for off-path scattering to affect this probe signal. A concentrated off-path disturbance could produce this wideangle scattering, but the NPM heating region is relatively diffuse with changes occurring gradually over the course of VLF wavelengths. To quantify this effect, Poulsen et al. [1990] presented a theoretical analysis of subionospheric VLF propagation in the presence of ionospheric disturbances and showed that for a Gaussian-shaped disturbance and simplified scattering geometries, an analytic expression exists for estimating the probe signal perturbation. Applying their Equation A13 with a heating region of $150 \mathrm{~km}$ effective radius as suggested by Rodriguez et al. [1994] for scattering geometries similar to the NPM-NLK-MI configuration, it can be shown that off-path scattering from the most intense heating region within $200 \mathrm{~km}$ of NPM falls off very rapidly as the distance to the probe signal pathway increases. For the distance of closest approach set to $100 \mathrm{~km}$, the predicted amplitude perturbation is $\sim 10^{-2} \mathrm{~dB}$. For $300 \mathrm{~km}$, the predicted perturbation falls to less than $\sim 10^{-4} \mathrm{~dB}$. For the $1750 \mathrm{~km}$ distance of closest approach for the NPM-NLKMI scattering configuration, the predicted perturbation is effectively zero. This supports the decision to ignore offpath scattering and focus the modeling efforts on the 2-D along-path scattering analysis.

[37] While the support of basic scattering theory may sufficiently justify the decision to focus on along-path scattering, we can also provide experimental justification by analyzing the arrival azimuth of the observed probe signal perturbations. The VLF receiver located at MI records two channels, each from one of a pair of orthogonal wire loop antennas. Typically, one of these antennas is oriented NorthSouth (NS) and one East-West (EW). For these experiments, however, the antennas were rotated slightly to align the EW antenna more directly toward NPM. Given the $77^{\circ}$ angle between NLK, NPM, and MI, the NPM signal arrives predominantly on the EW channel of the MI receiver while the NLK signal arrives predominantly on the NS channel. If the perturbation on the probe signal were due to offpath scattering from an ionospheric disturbance near NPM, then that perturbation should appear on the EW channel. However, the observed perturbations appeared on the NS channel and not on the EW channel. This suggests alongpath scatter as opposed to off-path scatter. We note that the MI receiver detects the local transverse magnetic field, so for this arrival azimuth analysis to hold we require that the scattering does not generate a large longitudinal magnetic field. A large longitudinal magnetic field could occur for scattering into high-order transverse electric modes, which is more common for scattering from intense, rapidly changing disturbances. Results from 2-D FDFD scattering analysis show that the perturbation to the transverse magnetic field is much larger than perturbation to the longitudinal magnetic field, so the probe signal and perturbation remain predominantly transverse magnetic and the arrival azimuth analysis discussed here remains valid.

[38] To put the arrival azimuth analysis into a more rigorous mathematical framework, we quantify the deviation in arrival azimuth, $\theta$, of the NLK-MI probe signal during each of the NPM keying sessions. For each keying session, we first estimate the probe signal arrival azimuth for samples during the times when NPM was off. Then we estimate the probe signal arrival azimuth for samples when NPM was on. Comparing these two values gives an estimate $\Delta \theta$ for the change in probe signal arrival azimuth due to scattering during this keying session. For along-path scatter, the scattering and probe signal perturbation arrive from the same direction as the signal itself. Thus, the perturbation should not change the probe signal arrival azimuth, and we should find $\Delta \theta=0^{\circ}$ for the case of along-path scatter. If the perturbation arrives from off-path, however, then arrival azimuth $\theta$ should change. For the NLK-MI probe signal, if the perturbation arrives from the direction of NPM with a magnitude of $0.8 \mathrm{fT}$ (average for the experimental observations), then we would expect to find $\Delta \theta=$ $0.03^{\circ}$. The distribution of $\Delta \theta$ is presented in Figure 15 for the 375 keying sessions for which a strong perturbation 


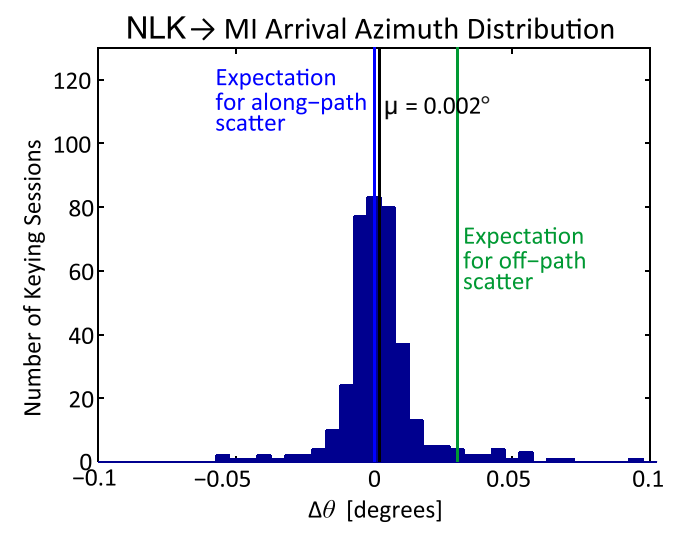

Figure 15. Distribution of arrival azimuth deviation $(\Delta \theta)$ of the NLK-MI probe signal for the 375 NPM keying sessions, which showed strong detection. The mean and standard deviation of this distribution are $0.002^{\circ}$ and $0.016^{\circ}$, respectively. The expected value for along-path scatter is marked in blue, and the expected value for off-path scatter is marked in green.

$(|\Delta A|>0.8 \mathrm{fT})$ exists. The expectation for along-path scatter is marked in blue $\left(\Delta \theta=0^{\circ}\right)$, and the expectation for off-path scatter is marked in green $\left(\Delta \theta=0.03^{\circ}\right)$. The distribution is clustered tightly near the expectation for along-path scatter, with a mean of $0.002^{\circ}$ and a standard deviation of $0.016^{\circ}$. So this experimental evidence, much like the theoretical analysis, suggests that the observed perturbations on the NLK-MI probe signal are due primarily to along-path scattering and that off-path scattering can be safely ignored.

\subsection{Model Self-Consistency}

[39] One more modeling component that warrants discussion is the lack of strict self-consistency in computing the electron heating, together with the decision to ignore changes to electron density. Wave propagation, electron heating, and electron density changes all influence one another. Therefore, any true calculation of one must solve for all three together. We must consider the error incurred by solving for each effect separately. At distances greater than $1000 \mathrm{~km}$ from the heating transmitter NPM, we estimate changes to the electron temperature of $\sim 1 \%$ or less. Application of an ionospheric chemistry model similar to that of Rodriguez and Inan [1994] shows that this $1 \%$ increase in electron temperature would lead to less than $0.5 \%$ change in electron density above $80 \mathrm{~km}$ altitude. The probe signal perturbation analysis showed these small changes produce on the order of $0.1 \%$ change to a subionospherically propagating VLF signal. So the feedback of these minor changes would be negligible for our purposes. We note that this analysis is sufficient to justify application of the model toward the key conclusion of this paper: extended lateral heating at great distances from the NPM transmitter are able to account for the perturbations observed on the NLK-MI probe signal. Recall that the NLK-MI probe signal pathway is never closer than $1750 \mathrm{~km}$ to the NPM transmitter and experiences electron temperature changes of $\sim 1 \%$ or less. We further note that probe signal scattering from changing electron densities should not even be considered when comparing to the experimental observations. While electron heating and cooling occur on the order of microseconds and milliseconds, respectively, electron density changes occur over tens of seconds [Glukhov et al., 1992; Rodriguez and Inan, 1994]. Since we key the NPM transmitter on/off at $0.1 \mathrm{~Hz}$ or faster and since the observed perturbation onset delay is less than $50 \mathrm{~ms}$, we can safely ignore the effects of changing electron densities.

[40] While it should have little effect on comparisons to the experimental observations or on the conclusions for heating at great distances, it is worth assessing feedback nearby the NPM transmitter where field intensities and ionospheric changes are strongest. There is as much as a $50 \%$ increase in electron temperature near NPM, which would produce a $\sim 20 \%$ electron density depletion at $80 \mathrm{~km}$ altitude [Rodriguez and Inan, 1994]. This significant density depletion would allow increased penetration of the VLF wave into the ionosphere, which would in turn produce increased heating and positive feedback. This effect was discussed by Rodriguez and Inan [1994], and they mention that the feedback would ultimately be inhibited by a maximum in the effective three-body electron attachment rate around $T_{e}=700 \mathrm{~K}$ [Tomko, 1981, p. 163]. While they do not compute the ultimate result of this feedback, it is likely dwarfed by the general ionospheric variability between a tenuous and a dense nighttime ionosphere. Still, a complete modeling of ionospheric heating overhead a powerful VLF transmitter should account for feedback between heating, density changes, and wave propagation. Heat flow may also become an important factor. Such a self-consistent model for heating of the lower ionosphere by VLF transmitters does not yet exist. It is also worth considering any possible connection between this lower ionosphere heating and the heating observed at $700 \mathrm{~km}$ altitude overhead VLF transmitters [Bell et al., 2011]. The higher-altitude heating is yet to be modeled, but Bell et al. [2011] suggest that high-altitude heating is most likely separate from the lower ionosphere heating and that it is due primarily to heating between the $E$ region and $700 \mathrm{~km}$ altitude. These changes to ionospheric electron temperatures and densities would have subsequent effects on VLF propagation, most notably upon the transionospheric propagation of VLF waves discussed recently by Cohen and Inan [2012], Cohen et al. [2012], and Graf et al. [2013].

\section{Conclusion}

[41] The controlled modulation of a ground-based VLF transmitter consistently perturbs the signal of a second VLF transmitter, showing a seasonal trend correlating with ionospheric electron density variation but no correlation with geomagnetic activity. Time signature analysis and laboratory testing of receiver equipment eliminates transmitterinduced precipitation of electron radiation and instrumental cross modulation as potential causes [Graf et al., 2011]. Experimental evidence suggests ionospheric heating by the modulated VLF transmitter to be the most probable cause. Analysis of the arrival azimuth of the perturbed signal leads to the conclusion that the perturbation is caused by extended lateral heating. Large-scale computational modeling corroborates ionospheric heating extending laterally to great distances within the Earth-ionosphere waveguide, with electron temperature increases of $\sim 1 \%$ extending up to $2000 \mathrm{~km}$ 
from the heating transmitter. Along-path scattering of the second VLF transmitter signal (the probe signal) from this extended lateral heating can account for the experimental observations. For a midlatitude, ground-based VLF transmitter at nighttime, $\sim 20 \%$ of the total radiated power penetrates through the ionosphere into the magnetosphere [Graf et al., 2013], 50\% contributes to ionospheric heating within $400 \mathrm{~km}$ lateral distance, and $~ 30 \%$ contributes to heating and attenuation at greater distances. Due to the efficiency with which VLF signals propagate to great distances in the Earth-ionosphere waveguide, the lateral extent of ionospheric heating due to powerful VLF transmitters is several thousand kilometers, significantly greater than previously recognized.

[42] Acknowledgments. This work has been supported by AFRL award FA9453-11-C-0011.

[43] Robert Lysak thanks Mark Golkowski and Michael Rietveld for their assistance in evaluating this paper.

\section{References}

Abel, B., and R. M. Thorne (1998a), Electron scattering loss in Earth's inner magnetosphere: 1. Dominant physical processes, J. Geophys. Res., 103(A2), 2385-2396.

Abel, B., and R. M. Thorne (1998b), Electron scattering loss in Earth's inner magnetosphere: 2. Sensitivity to model parameters, J. Geophys. Res., 103(A2), 2397-2407.

Bailey, V. A. (1938), On some effects caused in the ionosphere by electric waves-Part II, Philos. Mag., 26, 425-453.

Bailey, V. A., and D. F. Martyn (1934), Interaction of radio waves, Nature, 133, 218, doi:10.1038/133218a0.

Banks, P. (1966), Collision frequencies and energy transfer, Planet. Space Sci., 14, 1105-1122.

Barr, R., and P. Stubbe (1992), VLF heating of the lower ionosphere: Variation with magnetic latitude and electron density profile, Geophys. Res. Lett., 19, 1747-1750.

Barr, R., M. T. Rietveld, H. Kopka, and P. Stubbe (1984), Effect of a heated patch of auroral ionosphere on VLF-radio wave propagation, Nature, 309, 534-536, doi:10.1038/309534a0.

Bell, T. F., K. L. Graf, U. S. Inan, D. Piddyachiy, and M. Parrot (2011), DEMETER observations of ionospheric heating by powerful VLF transmitters, Geophys. Res. Lett., 38, L11103, doi:10.1029/2011GL047503.

Bilitza, D., and B. W. Reinisch (2008), International reference ionosphere 2007: Improvements and new parameters, Adv. Space Res., 42, 599-609, doi:10.1016/j.asr.2007.07.048.

Bittencourt, J. A. (2005), Fundamentals of Plasma Physics, 3rd ed., Springer, New York.

Budden, K. G. (1985), The Propagation of Radio Waves: The Theory of Radio Waves of Low Power in the Ionosphere and Magnetosphere, Cambridge Univ. Press, Cambridge, U. K.

Chevalier, M. W., and U. S. Inan (2006), A technique for efficiently modeling long-path propagation for use in both FDFD and FDTD, IEEE Antennas Wirel. Propag. Lett., 5, 525-528, doi:10.1109/LAWP.2006.887551.

Chevalier, M. W., W. B. Peter, U. S. Inan, T. F. Bell, and M. Spasojevic (2007), Remote sensing of ionospheric disturbances associated with energetic particle precipitation using the South Pole VLF beacon, J. Geophys. Res., 112, A11306, doi:10.1029/2007JA012425.

Chevalier, T. W., U. S. Inan, and T. F. Bell (2008), Terminal impedance and antenna current distribution of a VLF electric dipole in the inner magnetosphere, IEEE Trans. Antennas Propag., 56, 2454-2468, doi:10.1109/TAP.2008.927497.

Cohen, M. B., and U. S. Inan (2012), Terrestrial VLF transmitter injection into the magnetosphere, J. Geophys. Res., 117, A08310, doi:10.1029/2012JA017992.

Cohen, M. B., U. S. Inan, M. Gołkowski, and N. G. Lehtinen (2010a), On the generation of ELF/VLF waves for long-distance propagation via steerable HF heating of the lower ionosphere, J. Geophys. Res., 115, A07322, doi:10.1029/2009JA015170.

Cohen, M. B., U. S. Inan, and E. W. Paschal (2010b), Sensitive broadband ELF/VLF radio reception with the AWESOME instrument, IEEE Trans. Geosci. Remote Sens., 48, 3-17.

Cohen, M. B., N. G. Lehtinen, and U. S. Inan (2012), Models of ionospheric VLF absorption of powerful ground-based transmitters, Geophys. Res. Lett., 39, L24101, doi:10.1029/2012GL054437.
Cotts, B. R. T., U. S. Inan, and N. G. Lehtinen (2011), Longitudinal dependence of lightning-induced electron precipitation, J. Geophys. Res., 116, A10206, doi:10.1029/2011JA016581.

Crary, J. H. (1961), The effect of the Earth-ionosphere waveguide on whistlers, Tech. Rep. 9, Stanford Univ. Electron. Lab., Stanford, Calif.

Dalgarno, A., M. B. McElroy, M. H. Rees, and J. C. G. Walker (1968), The effect of oxygen cooling on ionospheric electron temperatures, Planet. Space Sci., 16, 1371-1380.

Fejer, J. A. (1970), Radio wave probing of the lower ionosphere by crossmodulation techniques, J. Atmos. Terr. Phys., 32, 597-607.

Foust, F. R., M. Spasojevic, T. F. Bell, and U. S. Inan (2011), Modeling scattering from lightning-induced ionospheric disturbances with the discontinuous Galerkin method, J. Geophys. Res., 116, A12301, doi:10.1029/2011JA016973.

Galejs, J. (1972), Ionospheric interaction of VLF radio waves, J. Atmos. Terr. Phys., 34, 421-436.

Ginzburg, V. L., and A. V. Gurevich (1960), Nonlinear phenomena in a plasma located in an alternating electromagnetic field, Sov. Phys. Uspekhi, 3, 115-146.

Glukhov, V. S., V. P. Pasko, and U. S. Inan (1992), Relaxation of transient lower ionospheric disturbances caused by lightning-whistler-induced electron precipitation bursts, J. Geophys. Res., 97(A11), 16,971-16,979.

Graf, K. L., U. S. Inan, D. Piddyachiy, P. Kulkarni, M. Parrot, and J. A. Sauvaud (2009), DEMETER observations of transmitter-induced precipitation of inner radiation belt electrons, J. Geophys. Res., 114, A07205, doi:10.1029/2008JA013949.

Graf, K. L., U. S. Inan, and M. Spasojevic (2011), Transmitterinduced modulation of subionospheric VLF signals: Ionospheric heating rather than electron precipitation, J. Geophys. Res., 116, A12313, doi:10.1029/2011JA016996.

Graf, K. L., N. G. Lehtinen, M. Spasojevic, M. B. Cohen, R. A. Marshall, and U. S. Inan (2013), Analysis of experimentally validated transionospheric attenuation estimates of VLF signals, J. Geophys. Res. Space Physics, 118, 2708-2720, doi:10.1002/jgra.50228.

Gurevich, A. V. (1978), Nonlinear Phenomena in the Ionosphere, 370 pp., Springer-Verlag, New York.

Helliwell, R. A. (1965), Whistlers and Related Ionospheric Phenomena, Stanford Univ. Press, Stanford, Calif.

Helliwell, R. A., J. P. Katsufrakis, and M. L. Trimpi (1973), Whistlerinduced amplitude perturbation in VLF propagation, J. Geophys. Res., 78(22), 4679-4688.

Huxley, L. G. H., and J. A. Ratcliffe (1949), A survey of ionospheric crossmodulation (wave interaction or Luxembourg effect), Proc. IEE - Part III: Radio Comm. Eng., 96(43), 433-440, doi:10.1049/pi-3.1949.0093.

Imhof, W. L., J. B. Reagan, H. D. Voss, E. E. Gaines, D. W. Datlowe, J. Mobilia, R. A. Helliwell, U. S. Inan, J. Katsufrakis, and R. G. Joiner (1983), Direct observation of radiation belt electrons precipitated by the controlled injection of VLF signals from a ground-based transmitter, Geophys. Res. Lett., 10(4), 361-364.

Inan, U. S. (1987), Gyroresonant pitch angle scattering by coherent and incoherent whistler mode waves in the magnetosphere, J. Geophys. Res., 92(A1), 127-142

Inan, U. S. (1990), VLF heating of the lower ionosphere, Geophys. Res. Lett., 17, 729-732.

Inan, U. S., H. C. Chang, R. A. Helliwell, W. L. Imhof, J. B. Reagan, and M. Walt (1985), Precipitation of radiation belt electrons by man-made waves: A comparison between theory and measurement, J. Geophys. Res., 90(A1), 359-369.

Inan, U. S., J. V. Rodriguez, S. Lev-Tov, and J. Oh (1992), Ionospheric modification with a VLF transmitter, Geophys. Res. Lett., 19, 2071-2074.

Inan, U. S., M. Golkowski, M. K. Casey, R. C. Moore, W. Peter, P. Kulkarni, P. Kossey, E. Kennedy, S. Meth, and P. Smit (2007), Subionospheric VLF observations of transmitter-induced precipitation of inner radiation belt electrons, Geophys. Res. Lett., 34, L02106, doi:10.1029/2006GL028494.

James, H. G. (1985), The ELF spectrum of artificially modulated D/Eregion conductivity, J. Atmos. Terr. Phys., 47(11), 1129-1142.

Jin, G., M. Spasojevic, M. B. Cohen, and U. S. Inan (2013), Utilizing nonlinear ELF generation in modulated ionospheric heating experiments for communications applications, Radio Sci., 48, 61-68, doi: $10.1002 /$ rds.20014.

Johnson, M. P., U. S. Inan, and D. S. Lauben (1999), Subionospheric VLF signatures of oblique (nonducted) whistler-induced precipitation, Geophys. Res. Lett., 26(23), 3569-3572.

Kim, K.-C., Y. Shprits, D. Subbotin, and B. Ni (2011), Understanding the dynamic evolution of the relativistic electron slot region including radial and pitch angle diffusion, J. Geophys. Res., 116, A10214, doi:10.1029/2011JA016684.

Koons, H. C., B. C. Edgar, and A. L. Vampola (1981), Precipitation of inner zone electrons by whistler mode waves from the VLF transmitters UMS and NWC, J. Geophys. Res., 86, 640-648. 
Kulkarni, P., U. S. Inan, T. F. Bell, and J. Bortnik (2008), Precipitation signatures of ground-based VLF transmitters, J. Geophys. Res., 113, A07214, doi:10.1029/2007JA012569.

Lehtinen, N. G., and U. S. Inan (2008), Radiation of ELF/VLF waves by harmonically varying currents into a stratified ionosphere with application to radiation by a modulated electrojet, J. Geophys. Res., 113, A06301, doi:10.1029/2007JA012911.

Lehtinen, N. G., and U. S. Inan (2009), Full-wave modeling of transionospheric propagation of VLF waves, Geophys. Res. Lett., 36, L03104, doi:10.1029/2008GL036535.

Lehtinen, N. G., R. A. Marshall, and U. S. Inan (2010), Full-wave modeling of "early" VLF perturbations caused by lightning electromagnetic pulses, J. Geophys. Res., 115, A00E40, doi:10.1029/ 2009JA014776.

Leyser, T. B., U. S. Inan, D. L. Carpenter, and M. L. Trimpi (1984), Diurnal variation of burst precipitation effects on subionospheric VLF/LF signal propagation near $l=2$, J. Geophys. Res., 89, 9139-9143.

Lyons, L. R., and D. J. Williams (1975), The quiet time structure of energetic (35-560 keV) radiation belt electrons, J. Geophys. Res., 80(7), 943-950.

Macmillan, S., and S. Maus (2005), International Geomagnetic Reference Field-The tenth generation, Earth Planets Space, 57, 1135-1140.

Marshall, R. A., and U. S. Inan (2010), Two-dimensional frequency domain modeling of lightning EMP-induced perturbations to VLF transmitter signals, J. Geophys. Res., 115, A00E29, doi:10.1029/2009JA014761.

Marshall, R. A., U. S. Inan, and T. W. Chevalier (2008), Early VLF perturbations caused by lightning EMP-driven dissociative attachment, Geophys. Res. Lett., 35, L21807, doi:10.1029/2008GL035358.

Marshall, R. A., R. T. Newsome, N. G. Lehtinen, N. Lavassar, and U. S Inan (2010), Optical signatures of radiation belt electron precipitation induced by ground-based VLF transmitters, J. Geophys. Res., 115 A08206, doi:10.1029/2010JA015394.

Maslin, N. M. (1975), Theory of the modifications imposed on the ionospheric plasma by a powerful radio wave reflected in the D or $\mathrm{E}$ region, Proc. R. Soc. London, Ser. A, 343, 109-131, doi:10.1098/rspa. 1975.0054.

Maslin, N. M. (1976), Estimating ionospheric cross-modulation, Proc. $R$. Soc. London, Ser. A, 351, 277-293, doi:10.1098/rspa.1976.0142.

Mentzoni, M. H., and R. V. Row (1963), Rotational excitation and electron relaxation in nitrogen, Phys. Rev., 130(6), 2312-2316.

Moore, R. C., U. S. Inan, T. F. Bell, and E. J. Kennedy (2007), ELF waves generated by modulated HF heating of the auroral electrojet and observed at a ground distance of $\sim 4400 \mathrm{~km}, J$. Geophys. Res., 112, A05309, doi:10.1029/2006JA012063.

Peter, W. B., and U. S. Inan (2004), On the occurrence and spatial extent of electron precipitation induced by oblique nonducted whistler waves, J. Geophys. Res., 109, A12215, doi:10.1029/2004JA010412.

Peter, W. B., and U. S. Inan (2007), A quantitative comparison of lightninginduced electron precipitation and VLF signal perturbations, J. Geophys. Res., 112, A12212, doi:10.1029/2006JA012165.
Picone, J. M., A. E. Hedin, D. P. Drob, and A. C. Aikin (2002), NRLMSISE00 empirical model of the atmosphere: Statistical comparisons and scientific issues, J. Geophys. Res., 107(A12), 1468, doi:10.1029/ 2002JA009430.

Poulsen, W. L., T. F. Bell, and U. S. Inan (1990), Three-dimensional modeling of subionospheric VLF propagation in the presence of localized D region perturbations associated with lightning, J. Geophys. Res., 95(A3), 2355-2366.

Rawer, K., D. Bilitza, and S. Ramakrishan (1978), Goals and status of the international reference ionosphere, Rev. Geophys., 16, 177-181.

Rietveld, M. T., H. Kopka, and P. Stubbe (1986), D-region characteristics deduced from pulsed ionospheric heating under auroral electrojet conditions, J. Atmos. Terr. Phys., 48(4), 311-326.

Rodriguez, J. V., and U. S. Inan (1994), Electron density changes in the nighttime $\mathrm{D}$ region due to heating by very-low-frequency transmitters, Geophys. Res. Lett., 21(2), 93-96.

Rodriguez, J. V., U. S. Inan, and T. F. Bell (1994), Heating of the nighttime D region by very low frequency transmitters, J. Geophys. Res., 99(A12), 23,329-23,338.

Schunk, R. W., and A. F. Nagy (1978), Electron temperatures in the F region of the ionosphere: Theory and observations, Rev. Geophys. Space Phys., 16(3), 355-399.

Starks, M. J., R. A. Quinn, G. P. Ginet, J. M. Albert, G. S. Sales, B. W. Reinisch, and P. Song (2008), Illumination of the plasmasphere by terrestrial very low frequency transmitters: Model validation, J. Geophys. Res., 113, A09320, doi:10.1029/2008JA013112.

Stubbe, P., and W. S. Varnum (1972), Electron energy transfer rates in the ionosphere, Planet. Space Sci., 20, 1121-1126.

Swamy, A. C. B. (1992), Equatorial electrojet parameters and the relevance of Electromagnetic Drifts (EMD) over Thumba, Astrophys. Space Sci. $191,203-211$.

Tao, X., J. Bortnik, and M. Friedrich (2010), Variance of transionospheric VLF wave power absorption, J. Geophys. Res., 115, A07303, doi:10.1029/2009JA015115.

Taranenko, Y. N., U. S. Inan, and T. F. Bell (1992), VLF-HF heating of the lower ionosphere and ELF wave generation, Geophys. Res. Lett., 19 , 61-64.

Taranenko, Y. N., U. S. Inan, and T. F. Bell (1993), The interaction with the lower ionosphere of electromagnetic pulses from lighting: Excitation of optical emissions, Geophys. Res. Lett., 20(23), 2675-2678.

Tellegen, B. D. H. (1933), Interaction between radiowaves?, Nature, 131, 840, doi:10.1038/131840a0.

Tomko, A. A. (1981), Nonlinear phenomena arising from radio wave heating of the lower ionosphere, Tech. Rep. PSU-IRL-SCI-470, The Pennsylvania State University.

Tomko, A. A., A. J. Ferraro, H. S. Lee, and A. P. Mitra (1980), A theoretical model of $D$-region ion chemistry modifications during high power radio wave heating, J. Atmos. Terr. Phys., 42, 275-285.

Vampola, A. L. (1977), VLF transmission induced slot electron precipitation, Geophys. Res. Lett., 4, 569-572. 